\title{
Wavelet frames for distributions; local and pointwise regularity
}

by

\author{
Hans Triebel (Jena)
}

\begin{abstract}
This paper deals with wavelet frames for a large class of distributions on euclidean $n$-space, including all compactly supported distributions. These representations characterize the global, local, and pointwise regularity of the distribution considered.
\end{abstract}

\section{INTRODUCTION}

Let $k$ be a non-negative $C^{\infty}$ function in $\mathbb{R}^{n}$ with compact support in

$$
\left\{y \in \mathbb{R}^{n}:|y|<1, y_{j}>0 \text { for all } j\right\}
$$

such that for some $J \in \mathbb{N}$,

$$
\sum_{m \in \mathbb{Z}^{n}} k\left(x-2^{-J} m\right)=1, \quad x \in \mathbb{R}^{n} .
$$

Let $k^{\beta}(x)=x^{\beta} k(x)$ for $x \in \mathbb{R}^{n}, \beta \in \mathbb{N}_{0}^{n}$, and let

$$
k^{\beta}(t, f)(x)=\int_{\mathbb{R}^{n}} k^{\beta}(y) f(x+t y) d y, \quad t>0, x \in \mathbb{R}^{n},
$$

be the corresponding local means with respect to the non-negative kernels $k^{\beta}(y)$ and $f \in S^{\prime}\left(\mathbb{R}^{n}\right)$. Let $\varphi_{0} \in S\left(\mathbb{R}^{n}\right)$ with

$$
\varphi_{0}=1 \text { if }|x| \leq 2^{-J} \text { and } \quad \varphi_{0}(x)=0 \text { if }|x| \geq 3 \cdot 2^{-J-1},
$$

and

$$
\varphi(x)=\varphi_{0}(x)-\varphi_{0}(2 x), \quad \varphi_{j}(x)=\varphi\left(2^{-j} x\right), \quad x \in \mathbb{R}^{n}, j \in \mathbb{N} .
$$

Then $\sum_{j=0}^{\infty} \varphi_{j}(x)=1$ is a dyadic resolution of unity in $\mathbb{R}^{n}$. Let

$$
\begin{gathered}
\omega \in S\left(\mathbb{R}^{n}\right), \quad \operatorname{supp} \omega \subset(-\pi, \pi)^{n}, \quad \omega(x)=1 \text { if }|x| \leq 2, \\
\omega^{\beta}(x)=\frac{i^{|\beta|} 2^{J|\beta|}}{(2 \pi)^{n} \beta !} x^{\beta} \omega(x), \quad \beta \in \mathbb{N}_{0}^{n},
\end{gathered}
$$

2000 Mathematics Subject Classification: 46E35, 42B35, 42C40. 
and

$$
\Omega^{\beta}(x)=\sum_{m \in \mathbb{Z}^{n}}\left(\omega^{\beta}\right)^{\vee}(m) e^{-i m x}, \quad x \in \mathbb{R}^{n}, \beta \in \mathbb{N}_{0}^{n} .
$$

We introduce the father wavelets $\Phi_{\mathrm{F}}^{\beta}$ and mother wavelets $\Phi_{\mathrm{M}}^{\beta}$ via their inverse Fourier transforms

$$
\left(\Phi_{\mathrm{F}}^{\beta}\right)^{\vee}(x)=\varphi_{0}(x) \Omega^{\beta}(x) \quad \text { and } \quad\left(\Phi_{\mathrm{M}}^{\beta}\right)^{\vee}(x)=\varphi(x) \Omega^{\beta}(x) .
$$

Obviously, $\Phi_{\mathrm{F}}^{\beta}$ and $\Phi_{\mathrm{M}}^{\beta}$ are elements of $S\left(\mathbb{R}^{n}\right)$; furthermore, they are entire analytic functions with

$$
\int_{\mathbb{R}^{n}} \Phi_{\mathrm{M}}^{\beta}(x) x^{\alpha} d x=0 \quad \text { for all } \alpha \in \mathbb{N}_{0}^{n} .
$$

Then, for example, any compactly supported distribution $f \in S^{\prime}\left(\mathbb{R}^{n}\right)$ can be represented as

$$
f=2^{J n} \sum_{\beta, j, m} k^{\beta}\left(2^{-j+J}, f\right)\left(2^{-j} m\right) \Phi^{\beta}\left(2^{j} x-m\right),
$$

with unconditional convergence in $S^{\prime}\left(\mathbb{R}^{n}\right)$, where $\beta \in \mathbb{N}_{0}^{n}, j \in \mathbb{N}_{0}, m \in \mathbb{Z}^{n}$, and

$$
\Phi^{\beta}\left(2^{j} x-m\right)= \begin{cases}\Phi_{\mathrm{F}}^{\beta}(x-m) & \text { if } j=0 \\ \Phi_{\mathrm{M}}^{\beta}\left(2^{j} x-m\right) & \text { if } j \in \mathbb{N} .\end{cases}
$$

This wavelet representation is optimal in several respects. First, let $s<0$, $1<p \leq \infty$, and let $B_{p}^{s}\left(\mathbb{R}^{n}\right)=B_{p p}^{s}\left(\mathbb{R}^{n}\right)$ be the corresponding special Besov spaces. Then

$$
f \in B_{p}^{s}\left(\mathbb{R}^{n}\right) \text { if, and only if, }\left\{2^{j(s-n / p)} k^{\beta}\left(2^{-j+J}, f\right)\left(2^{-j} m\right)\right\}_{\beta, j, m} \in \ell_{p}
$$
(equivalent norms). Secondly, (1.1) reflects both the global and local behaviour of $f$. Let $B(y, r)$ be the ball centred at $y \in \mathbb{R}^{n}$ and of radius $r>0$. Let $x^{0} \in \mathbb{R}^{n}, K \in \mathbb{N}$, and let $\sum_{\beta, j, m}^{K, x^{0}}$ be that part of the sum in (1.1) with

$$
B\left(2^{-j} m, 2^{-j+J}\right) \cap B\left(x^{0}, 2^{-K+1}\right) \neq \emptyset .
$$

Then for $x \in B\left(x^{0}, 2^{-K-1}\right)$,

$$
f=2^{J n} \sum_{\beta, j, m}^{K, x^{0}} k^{\beta}\left(2^{-j+J}, f\right)\left(2^{-j} m\right) \Phi^{\beta}\left(2^{j} x-m\right) \bmod C^{\infty}
$$

gives a satisfactory local description of $f$ in $B\left(x^{0}, 2^{-K-1}\right)$ modulo a $C^{\infty}$ function (this means that the remainder term is a $C^{\infty}$ function in $B\left(x^{0}, 2^{-K-1}\right)$ ). In particular restricting the $\ell_{p}$-sum in (1.2) to the respective terms in (1.3) one may ask whether $s$ can be improved to $s_{K}$ with $s<s_{K}<0$, so that $f \in B_{p}^{s_{K}}\left(B\left(x^{0}, 2^{-K-1}\right)\right)$. If $K \rightarrow \infty$ and $s_{K} \uparrow s_{\infty}<0$ then one gets a definitive information concerning the local regularity of $f$ in $B\left(x^{0}, 2^{-K-1}\right)$ 
and pointwise regularity at $x^{0}$ in terms of Besov spaces (recall that the Hölder-Zygmund spaces $\mathcal{C}^{s}=B_{\infty}^{s}$ are covered as special cases). This theory can be extended to $s \geq 0,1<p \leq \infty$. For this purpose one has to replace the non-negative kernels $k^{\beta}(y)$ by oscillating kernels $k_{L}^{\beta}(y)=(-\Delta)^{L} k^{\beta}(y)$ as the simplest choice where $\Delta$ is the Laplacian and $L \in \mathbb{N}_{0}$, hence

$$
k_{L}^{\beta}(t, f)(x)=\int_{\mathbb{R}^{n}} k_{L}^{\beta}(y) f(x+t y) d y, \quad t>0, x \in \mathbb{R}^{n} .
$$

One gets representations of type (1.1) with modified (father and mother) wavelets. We shall give an explicit calculation of all functions and constants. The local and pointwise regularity theory can now be extended to $s \in \mathbb{R}$. For example one has again an explicit local description of type (1.3).

It is the main aim of this paper to present this roughly outlined theory in detail, complemented by quarkonial decompositions of functions

$$
f \in B_{p}^{s}\left(\mathbb{R}^{n}\right), \quad 1 \leq p \leq \infty, s>0 .
$$

These functions can be represented as

$$
f=\sum_{\beta, j, m} 2^{j n}\left(f, \Phi^{\beta}\left(2^{j} \cdot-m\right)\right) k^{\beta}\left(2^{j-J} x-2^{-J} m\right),
$$

with absolute convergence in $L_{p}\left(\mathbb{R}^{n}\right)$, where $\Phi^{\beta}$ and $k^{\beta}$ have the above meaning and where the coefficients of $k^{\beta}\left(2^{j-J} x-2^{-J} m\right)$ satisfy the counterpart of (1.2). First we deal in Subsection 2.2 with these quarkonial decompositions adapting the corresponding theory of [14, Sections 2 and 3], where we considered the general spaces $B_{p q}^{s}\left(\mathbb{R}^{n}\right), F_{p q}^{s}\left(\mathbb{R}^{n}\right)$ for all admitted parameters. Dualizing the outcome we get afterwards in Subsection 2.3 the outlined representations (1.1), (1.3) for spaces $B_{p}^{s}\left(\mathbb{R}^{n}\right)$ with $1<p \leq \infty, s<0$. This part might be considered as the continuation of our considerations in [16], where we studied fractal characteristics of Radon measures in terms of function spaces. In Subsection 2.4 we deal with the extension of these assertions to functions $f$ as in (1.5) (now $p>1$ ). Finally we characterize in Subsection 2.5 the pointwise regularity of distributions in terms of local means. We begin with Subsection 2.1 where we collect a few definitions and preliminaries. Longer proofs are shifted to Section 3.

Wavelets, wavelet frames and, in particular, wavelet bases attracted a lot of attention, mostly in $L_{2}\left(\mathbb{R}^{n}\right)$. There are several extensions to $L_{p}\left(\mathbb{R}^{n}\right)$, Sobolev spaces $H_{p}^{s}\left(\mathbb{R}^{n}\right)$, Hölder-Zygmund spaces $\mathcal{C}^{s}\left(\mathbb{R}^{n}\right)$, and Besov spaces $B_{p q}^{s}\left(\mathbb{R}^{n}\right)$, for $1<p<\infty, 1 \leq q \leq \infty, s \in \mathbb{R}$. We refer to [7, Chapter 6]; [1, Chapter 9]; [18, Chapter 9]; [6, Chapter 9]. Further generalizations of wavelet representations to the more general spaces $B_{p q}^{s}\left(\mathbb{R}^{n}\right)$ and $F_{p q}^{s}\left(\mathbb{R}^{n}\right)$ may be found in $[2$, Section 7$]$ and $[9,2.3 .3]$. Usually one asks to what extent the Fourier coefficients with respect to a wavelet basis of a given function or distribution $f$ characterize $f$ as an element of one of the above 
spaces. This results in a global assertion which does not say very much about the, possibly improved, local regularity of $f$ or regularity at a given point $x^{0} \in \mathbb{R}^{n}$. The adaptation of wavelet techniques to study local regularity has been considered in detail in [5], [8], and more recently in [3], [4]. Although our paper must be seen as a contribution to this field, it has little in common with the techniques in the cited books and papers. The main point of our approach is the combination of the wavelet philosophy with the Taylorexpansion philosophy, that is,

$$
f(x) \rightarrow f\left(2^{j} x-m\right) \quad \text { with } \quad f(x) \rightarrow x^{\beta} f(x), \quad \text { respectively, }
$$

for $j \in \mathbb{N}_{0}, m \in \mathbb{Z}^{n}$, and $\beta \in \mathbb{N}_{0}^{n}$. Then one gets comparatively simple explicit wavelet frames which reflect simultaneously global and local behaviour. It turns out that the additional summation over $\beta \in \mathbb{N}_{0}^{n}$, complementing the standard wavelet summations over $j \in \mathbb{N}_{0}, m \in \mathbb{Z}^{n}$, is rather harmless. For example, the norms of the $\beta$-terms in (1.1) in some spaces $B_{p}^{s}\left(\mathbb{R}^{n}\right)$ are always of exponential decay of type $C_{\varrho} 2^{-\varrho|\beta|}$ where $\varrho \geq 0$ can be prescribed at the expense of some constants $C_{\varrho}$ which are independent of $\beta$. In connection with quarkonial decompositions of type (1.6), in Subsection 2.2 and in the related proofs in Subsection 3.1 we give a detailed account of the influence of the summation over $\beta \in \mathbb{N}_{0}^{n}$ and the role of $\varrho \geq 0$. But afterwards it is clear what can be expected and we will not stress this point. Furthermore, as will be described in 2.5, in order to characterize local and pointwise regularity one needs only local means of type (1.4) with $\beta=0$.

\section{DEFINITIONS, RESULTS, COMMENTS}

\subsection{Basic notation and function spaces}

2.1.1. Basic notation. We use standard notation. Let $\mathbb{N}$ be the collection of all natural numbers and $\mathbb{N}_{0}=\mathbb{N} \cup\{0\}$. Let $\mathbb{R}^{n}$ be euclidean $n$-space, where $n \in \mathbb{N}$. Put $\mathbb{R}=\mathbb{R}^{1}$. As usual, $\mathbb{Z}$ is the collection of all integers. Furthermore, $\mathbb{Z}^{n}$, where $n \in \mathbb{N}$, denotes the lattice of all points $m=\left(m_{1}, \ldots, m_{n}\right) \in \mathbb{R}^{n}$ with $m_{j} \in \mathbb{Z}$. The set $\mathbb{N}_{0}^{n}$ of all multi-indices consists of all points $\beta=$ $\left(\beta_{1}, \ldots, \beta_{n}\right) \in \mathbb{R}^{n}$ with $\beta_{j} \in \mathbb{N}_{0}$. We use with preference some letters for elements of the indicated sets:

$$
\mathbb{N}, \mathbb{N}_{0}: j, J ; \quad \mathbb{Z}^{n}: l, m ; \quad \mathbb{N}_{0}^{n}: \alpha, \beta .
$$

Let $S\left(\mathbb{R}^{n}\right)$ be the Schwartz space of all complex-valued, rapidly decreasing, infinitely differentiable functions on $\mathbb{R}^{n}$. By $S^{\prime}\left(\mathbb{R}^{n}\right)$ we denote its topological dual, the space of all tempered distributions on $\mathbb{R}^{n}$. Furthermore, $L_{p}\left(\mathbb{R}^{n}\right)$ with $0<p \leq \infty$ is the standard quasi-Banach space of all complex-valued functions with respect to the Lebesgue measure, quasi-normed by 


$$
\left\|f \mid L_{p}\left(\mathbb{R}^{n}\right)\right\|=\left(\int_{\mathbb{R}^{n}}|f(x)|^{p} d x\right)^{1 / p}
$$

with the usual modification if $p=\infty$. If $\varphi \in S\left(\mathbb{R}^{n}\right)$ then

$$
\widehat{\varphi}(\xi)=(F \varphi)(\xi)=(2 \pi)^{-n / 2} \int_{\mathbb{R}^{n}} e^{-i x \xi} \varphi(x) d x, \quad \xi \in \mathbb{R}^{n},
$$

denotes the Fourier transform of $\varphi$. As usual, $F^{-1} \varphi$ or $\varphi^{\vee}$ stands for the inverse Fourier transform, given by the right-hand side of (2.1) with $i$ in place of $-i$. Here $x \xi$ denotes the scalar product in $\mathbb{R}^{n}$. Both $F$ and $F^{-1}$ are extended to $S^{\prime}\left(\mathbb{R}^{n}\right)$ in the standard way.

2.1.2. Function spaces. Let $J \in \mathbb{N}, \varphi_{0} \in S\left(\mathbb{R}^{n}\right)$ with

$$
\varphi_{0}(x)=1 \text { if }|x| \leq 2^{-J} \text { and } \varphi_{0}(x)=0 \text { if }|x| \geq 3 \cdot 2^{-J-1} \text {, }
$$

and

$$
\varphi(x)=\varphi_{0}(x)-\varphi_{0}(2 x), \quad \varphi_{j}(x)=\varphi\left(2^{-j} x\right), \quad x \in \mathbb{R}^{n}, j \in \mathbb{N} .
$$

Then $\sum_{j=0}^{\infty} \varphi_{j}(x)=1$ with $x \in \mathbb{R}^{n}$ is a dyadic resolution of unity. Recall that $\left(\varphi_{j} \widehat{f}\right)^{\vee}$ is an entire analytic function on $\mathbb{R}^{n}$ for any $f \in S^{\prime}\left(\mathbb{R}^{n}\right)$. In particular, $\left(\varphi_{j} \widehat{f}\right)^{\vee}(x)$ makes sense pointwise.

Definition 1 . Let $0<p \leq \infty$ and $s \in \mathbb{R}$. Let $\left\{\varphi_{j}\right\}$ be the above dyadic resolution of unity in $\mathbb{R}^{n}$. For $f \in S^{\prime}\left(\mathbb{R}^{n}\right)$, let

$$
\left\|f \mid B_{p}^{s}\left(\mathbb{R}^{n}\right)\right\|_{\varphi}=\left(\sum_{j=0}^{\infty} 2^{j s p}\left\|\left(\varphi_{j} \widehat{f}\right)^{\vee} \mid L_{p}\left(\mathbb{R}^{n}\right)\right\|^{p}\right)^{1 / p}
$$

with the usual modification if $p=\infty$. Then

$$
B_{p}^{s}\left(\mathbb{R}^{n}\right)=\left\{f \in S^{\prime}\left(\mathbb{R}^{n}\right):\left\|f \mid B_{p}^{s}\left(\mathbb{R}^{n}\right)\right\|_{\varphi}<\infty\right\} .
$$

REMARK 1. These are special cases of the well-known Besov spaces $B_{p q}^{s}\left(\mathbb{R}^{n}\right)$ where now $q=p$. The theory of these spaces and of the spaces $F_{p q}^{s}\left(\mathbb{R}^{n}\right)$ has been developed in detail in [11], [12]. We remark that the quasi-norms in (2.4) for admitted functions $\varphi_{j}$ are equivalent to each other. In particular, $B_{p}^{s}\left(\mathbb{R}^{n}\right)$ are quasi-Banach spaces (Banach spaces if $p \geq 1$ ) which are independent of $\left\{\varphi_{j}\right\}$. This justifies our omission of the subscript $\varphi$ of $(2.4),(2.5)$ in what follows. In this paper we are interested in individual functions and distributions $f \in S^{\prime}\left(\mathbb{R}^{n}\right)$ rather than in the scales of the function spaces $B_{p q}^{s}\left(\mathbb{R}^{n}\right)$ and $F_{p q}^{s}\left(\mathbb{R}^{n}\right)$. But we wish to emphasize that a substantial part of the theory presented in this paper, and formulated in terms of the spaces $B_{p}^{s}\left(\mathbb{R}^{n}\right)$, can be extended to the scales $B_{p q}^{s}\left(\mathbb{R}^{n}\right)$ and $F_{p q}^{s}\left(\mathbb{R}^{n}\right)$. We remark that

$$
\mathcal{C}^{s}\left(\mathbb{R}^{n}\right)=B_{\infty}^{s}\left(\mathbb{R}^{n}\right), \quad s \in \mathbb{R},
$$

are the Hölder-Zygmund spaces. 
Proposition 1. There is a number $J_{0} \in \mathbb{N}_{0}$ with the following property. Let $J \in \mathbb{N}_{0}$ with $J \geq J_{0}$ in (2.2), (2.3), let $1 \leq p \leq \infty$ and let $s \in \mathbb{R}$. Then

$$
\left(\sum_{j=0}^{\infty} \sum_{m \in \mathbb{Z}^{n}} 2^{j(s-n / p) p}\left|\left(\varphi_{j} \widehat{f}\right)^{\vee}\left(2^{-j} m\right)\right|^{p}\right)^{1 / p} \sim\left\|f \mid B_{p}^{s}\left(\mathbb{R}^{n}\right)\right\|
$$

(with the usual modification if $p=\infty$ ), where the equivalence constants are independent of $J, p, s$, and $f$.

REMARK 2. In particular, for given $f \in S^{\prime}\left(\mathbb{R}^{n}\right)$ the left-hand side of (2.6) is finite if, and only if, $f \in B_{p}^{s}\left(\mathbb{R}^{n}\right)$. This follows from (2.4) and the equivalence assertions in $[13,(14.56)$, p. 102], where we referred in turn to [11, pp. 19-22]. There one also finds a discussion of the optimal choice of $J_{0}$ (again with references to the literature, especially to [10]). The fact that $J_{0}$ is independent of $s$ is obvious; that it is independent of $p$ with $1 \leq p \leq \infty$ is convenient for us, but not important. It simplifies some formulations. In particular, the above proposition can be extended to $0<p \leq \infty$ and $J \geq J_{0}(p)$. But this will not be needed in what follows.

\subsection{Decompositions and wavelets}

2.2.1. Some preparations, basic wavelets. In [14, Sections 2 and 3], we developed the constructive theory of the quarkonial (or subatomic) decompositions of the spaces $B_{p q}^{s}\left(\mathbb{R}^{n}\right)$ and $F_{p q}^{s}\left(\mathbb{R}^{n}\right)$ for the full range of the admitted parameters. Now we restrict our attention to the spaces $B_{p}^{s}\left(\mathbb{R}^{n}\right)$ with $s>0$ and $1 \leq p \leq \infty$. But somewhat in contrast to [14] we now need more detailed information concerning generating functions (wavelets) and some constants. First we fix some notation. Let

$$
\mathbb{R}_{++}^{n}=\left\{y \in \mathbb{R}^{n}: y=\left(y_{1}, \ldots, y_{n}\right), y_{j}>0 \text { for all } j\right\}
$$

and let $k$ be a non-negative $C^{\infty}$ function in $\mathbb{R}^{n}$ with

$$
\text { supp } k \subset\left\{y \in \mathbb{R}^{n}:|y|<1\right\} \cap \mathbb{R}_{++}^{n},
$$

and for some $J \in \mathbb{N}$,

$$
\sum_{m \in \mathbb{Z}^{n}} k\left(x-2^{-J} m\right)=1, \quad x \in \mathbb{R}^{n} .
$$

In addition we always assume $J \geq J_{0}$, where $J_{0}$ is as in Proposition 1 . Recall $x^{\beta}=x_{1}^{\beta_{1}} \ldots x_{n}^{\beta_{n}}$ where $x=\left(x_{1}, \ldots, x_{n}\right) \in \mathbb{R}^{n}$ and $\beta \in \mathbb{N}_{0}^{n}$. Then

$$
k^{\beta}(x)=x^{\beta} k(x) \geq 0, \quad x \in \mathbb{R}^{n}, \beta \in \mathbb{N}_{0}^{n} .
$$

Let

$$
\lambda=\left\{\lambda_{j m}^{\beta} \in \mathbb{C}: j \in \mathbb{N}_{0}, m \in \mathbb{Z}^{n}, \beta \in \mathbb{N}_{0}^{n}\right\}
$$


For $s \in \mathbb{R}, 1 \leq p \leq \infty$, and $\varrho \geq 0$, we put

$$
\left\|\lambda \mid \ell_{p}\right\|_{\varrho, s}=\left(\sum_{\beta \in \mathbb{N}_{0}^{n}} \sum_{j=0}^{\infty} \sum_{m \in \mathbb{Z}^{n}} 2^{\varrho|\beta| p+j(s-n / p) p}\left|\lambda_{j m}^{\beta}\right|^{p}\right)^{1 / p}
$$

(with the usual modification if $p=\infty$ ). We abbreviate

$$
\sum_{\beta, j, m}=\sum_{\beta \in \mathbb{N}_{0}^{n}} \sum_{j=0}^{\infty} \sum_{m \in \mathbb{Z}^{n}} .
$$

Let

$$
\omega \in S\left(\mathbb{R}^{n}\right), \quad \operatorname{supp} \omega \subset(-\pi, \pi)^{n}, \quad \omega(x)=1 \text { if }|x| \leq 2,
$$

and let

$$
\omega^{\beta}(x)=\frac{i^{|\beta|} 2^{J|\beta|}}{(2 \pi)^{n} \beta !} x^{\beta} \omega(x) \quad \text { for } x \in \mathbb{R}^{n} \text { and } \beta \in \mathbb{N}_{0}^{n} .
$$

Recall $|\beta|=\beta_{1}+\ldots+\beta_{n}$ and $\beta !=\beta_{1}$ ! $\ldots \beta_{n}$ !. Let

$$
\Omega^{\beta}(x)=\sum_{m \in \mathbb{Z}^{n}}\left(\omega^{\beta}\right)^{\vee}(m) e^{-i m x}, \quad x \in \mathbb{R}^{n} .
$$

Definition 2. Let $\varphi_{0}$ and $\varphi$ be given by (2.2), (2.3). Let $\beta \in \mathbb{N}_{0}^{n}$. Then the father wavelets $\Phi_{\mathrm{F}}^{\beta}(x)$ and the mother wavelets $\Phi_{\mathrm{M}}^{\beta}(x)$ are given by

$$
\begin{aligned}
\left(\Phi_{\mathrm{F}}^{\beta}\right)^{\vee}(\xi) & =\varphi_{0}(\xi) \Omega^{\beta}(\xi), & & \xi \in \mathbb{R}^{n}, \\
\left(\Phi_{\mathrm{M}}^{\beta}\right)^{\vee}(\xi) & =\varphi(\xi) \Omega^{\beta}(\xi), & & \xi \in \mathbb{R}^{n} .
\end{aligned}
$$

Remark 3. Since $\omega^{\beta} \in S\left(\mathbb{R}^{n}\right)$ it follows that $\left(\Phi_{\mathrm{F}}^{\beta}\right)^{\vee},\left(\Phi_{\mathrm{M}}^{\beta}\right)^{\vee}$, and hence also $\Phi_{\mathrm{F}}^{\beta}, \Phi_{\mathrm{M}}^{\beta}$, are elements of $S\left(\mathbb{R}^{n}\right)$. Furthermore, $\Phi_{\mathrm{F}}^{\beta}$ and $\Phi_{\mathrm{M}}^{\beta}$ are entire analytic functions with vanishing moments of all orders, and

$$
\int_{\mathbb{R}^{n}} \Phi_{\mathrm{M}}^{\beta}(\xi) \xi^{\alpha} d \xi=0, \quad \alpha \in \mathbb{N}_{0}^{n} .
$$

This follows from

$$
\operatorname{supp} \varphi \subset\left\{x \in \mathbb{R}^{n}: 2^{-J-1} \leq|x| \leq 3 \cdot 2^{-J-1}\right\} .
$$

We have

$$
\begin{aligned}
& \Phi_{\mathrm{F}}^{\beta}(x)=\sum_{m \in \mathbb{Z}^{n}}\left(\omega^{\beta}\right)^{\vee}(m) \widehat{\varphi}_{0}(x+m), \quad x \in \mathbb{R}^{n}, \\
& \Phi_{\mathrm{M}}^{\beta}(x)=\sum_{m \in \mathbb{Z}^{n}}\left(\omega^{\beta}\right)^{\vee}(m) \widehat{\varphi}(x+m), \quad x \in \mathbb{R}^{n} .
\end{aligned}
$$


2.2.2. Quarkonial decompositions. Let $\Phi_{\mathrm{F}}^{\beta}$ and $\Phi_{\mathrm{M}}^{\beta}$ be given by Definition 2. We introduce the wavelets

$$
\Phi^{\beta}\left(2^{j} x-m\right)= \begin{cases}\Phi_{\mathrm{F}}^{\beta}(x-m) & \text { if } j=0 \\ \Phi_{\mathrm{M}}^{\beta}\left(2^{j} x-m\right) & \text { if } j \in \mathbb{N} .\end{cases}
$$

According to the dual pairing $\left(S\left(\mathbb{R}^{n}\right), S^{\prime}\left(\mathbb{R}^{n}\right)\right)$ we put, for given $f \in S^{\prime}\left(\mathbb{R}^{n}\right)$,

$$
\lambda_{j m}^{\beta}(f)=2^{j n}\left(f, \Phi^{\beta}\left(2^{j} \cdot-m\right)\right), \quad j \in \mathbb{N}_{0}, m \in \mathbb{Z}^{n}, \beta \in \mathbb{N}_{0}^{n} .
$$

Finally, let

$$
B_{p}^{0+}\left(\mathbb{R}^{n}\right)=\bigcup_{s>0} B_{p}^{s}\left(\mathbb{R}^{n}\right), \quad 1 \leq p \leq \infty .
$$

Theorem 1. Let $1 \leq p \leq \infty, s>0$, and $\varrho \geq 0$.

(i) $f \in S^{\prime}\left(\mathbb{R}^{n}\right)$ is an element of $B_{p}^{s}\left(\mathbb{R}^{n}\right)$ if, and only if, it can be represented as

$$
f=\sum_{\beta, j, m} \lambda_{j m}^{\beta} k^{\beta}\left(2^{j-J} x-2^{-J} m\right), \quad x \in \mathbb{R}^{n},
$$

with $\left\|\lambda \mid \ell_{p}\right\|_{\varrho, s}<\infty$ and absolute convergence in $L_{p}\left(\mathbb{R}^{n}\right)$. Furthermore,

$$
\left\|f\left|B_{p}^{s}\left(\mathbb{R}^{n}\right)\|\sim \inf \| \lambda\right| \ell_{p}\right\|_{\varrho, s},
$$

where the infimum is taken over all admissible representations (2.25). as

(ii) Let $\lambda_{j m}^{\beta}(f)$ be given by $(2.24)$. Then $f \in B_{p}^{0+}\left(\mathbb{R}^{n}\right)$ can be represented

$$
f=\sum_{\beta, j, m} \lambda_{j m}^{\beta}(f) k^{\beta}\left(2^{j-J} x-2^{-J} m\right)
$$

with absolute convergence in $L_{p}\left(\mathbb{R}^{n}\right)$. In addition, $f \in B_{p}^{s}\left(\mathbb{R}^{n}\right)$ if, and only if, $\left\|\lambda(f) \mid \ell_{p}\right\|_{\varrho, s}<\infty$.

(iii) Let $f \in B_{p}^{s}\left(\mathbb{R}^{n}\right)$. Then (2.27) is an optimal representation, in the sense that

$$
\left\|f\left|B_{p}^{s}\left(\mathbb{R}^{n}\right)\|\sim\| \lambda(f)\right| \ell_{p}\right\|_{\varrho, s},
$$

where the equivalence constants are independent of $f$.

REMARK 4. Part (i) is a specification and modification of [14, Theorem 2.9 , pp. 15-16]. We give a modified proof adapting the technique developed in [14] to the above situation. In particular we are now interested in the explicit representation (2.27) with (2.28). The optimal coefficients $\lambda_{j m}^{\beta}(f)$ given by (2.24) depend linearly on $f$. Then $(2.27),(2.28)$ is called a frame representation. For given $\varrho \geq 0$ the equivalence constants in (2.28) depend on $\varrho$. In a more general context we discussed this point in detail in [14, especially $2.10,2.11$, pp. 21-23]. It turns out that the left-hand side in (2.26) can be estimated from above and from below by the right-hand side with the help of the constants $c_{1} 2^{K \varrho}$ and $c_{2} 2^{-K \varrho}$, respectively, where $c_{1}>0$, 
$c_{2}>0, K>0$ are some constants which are independent of $\varrho$. Later on in the proofs we use simplified arguments which result in the inequality (3.12) below for the frame coefficients $\lambda_{j m}^{\beta}(f)$. This will prove that for any $\varrho>0$ there is a constant $c(\varrho)$ such that for all $\beta \in \mathbb{N}_{0}^{n}$,

$$
\left(\sum_{j=0}^{\infty} \sum_{m \in \mathbb{Z}^{n}} 2^{j(s-n / p) p}\left|\lambda_{j m}^{\beta}(f)\right|^{p}\right)^{1 / p} \leq c(\varrho) 2^{-\varrho|\beta|}\left\|f \mid B_{p}^{s}\left(\mathbb{R}^{n}\right)\right\|
$$

(usual modification if $p=\infty$ ). Using (3.11) below one can estimate $c(\varrho)$ explicitly. But this will not be done. In the following we are mainly interested in the case $\varrho=0$.

\subsection{Wavelet frames for distributions}

2.3.1. Preliminaries. Let $0<p \leq \infty$. Then

$$
B_{p}^{-\infty}\left(\mathbb{R}^{n}\right)=\bigcup_{s<0} B_{p}^{s}\left(\mathbb{R}^{n}\right) .
$$

If $0<p_{1} \leq p_{2} \leq \infty$, then by well-known embedding theorems we have

$$
B_{p_{1}}^{-\infty}\left(\mathbb{R}^{n}\right) \subset B_{p_{2}}^{-\infty}\left(\mathbb{R}^{n}\right) \subset B_{\infty}^{-\infty}\left(\mathbb{R}^{n}\right)=\mathcal{C}^{-\infty}\left(\mathbb{R}^{n}\right),
$$

where the latter notation reminds of the Hölder-Zygmund scale. If $f \in$ $D^{\prime}\left(\mathbb{R}^{n}\right)$ has a compact support then $f$ belongs to any space $B_{p}^{-\infty}\left(\mathbb{R}^{n}\right)$. It is the main aim of this subsection to construct for $f \in \mathcal{C}^{-\infty}\left(\mathbb{R}^{n}\right)$ (and hence, in particular, for any compactly supported distribution in $\mathbb{R}^{n}$ ) universal wavelet representations

$$
f=\sum_{\beta, j, m} \lambda_{j m}^{\beta} \Phi^{\beta}\left(2^{j} x-m\right),
$$

where $\Phi^{\beta}\left(2^{j} x-m\right)$ are the wavelets as in (2.23). We have used the abbreviation (2.13). We begin with a discussion of representations of type (2.31). Let $1 \leq p \leq \infty$ and $s<0$. By the properties mentioned in Remark 3 it follows that for fixed $\beta \in \mathbb{N}_{0}^{n}$,

$$
\left\{2^{-j(s-n / p)} \Phi^{\beta}\left(2^{j} x-m\right): j \in \mathbb{N}_{0}, m \in \mathbb{Z}^{n}\right\}, \quad \beta \in \mathbb{N}_{0}^{n},
$$

are normalized molecules in $B_{p}^{s}\left(\mathbb{R}^{n}\right)$ according to $[2$, Section 5 , especially p. 48], combined with our usual normalization. In addition, the influence of $\beta$ in (2.31) is harmless and of the same type as in (2.29), where $\varrho \geq 0$ can be prescribed and where $\lambda_{j m}^{\beta}$ are optimal coefficients. The arguments are the same as there and as in the underlying proof in Step 1 of 3.1. But the shortest way to say what is meant by (2.31) is to lift $f$ and its representation by

$$
g=\left(\mathrm{id}+(-\Delta)^{L}\right)^{-1} f=\sum_{\beta, j, m} \lambda_{j m}^{\beta}\left(\mathrm{id}+(-\Delta)^{L}\right)^{-1} \Phi^{\beta}\left(2^{j} x-m\right)
$$


with $\sigma=2 L+s>0$. We discuss this type of lifting in detail in 2.4. It turns out that $(2.33)$ is a (slightly perturbed) wavelet representation. One can apply Theorem 1 and Remark 4 to $B_{p}^{\sigma}\left(\mathbb{R}^{n}\right)$. Hence (2.33) converges absolutely in $L_{p}\left(\mathbb{R}^{n}\right)$, and consequently unconditionally with respect to any rearrangement and any summation of subsums. Application of id $+(-\Delta)^{L}$ to (2.33) shows that $(2.31)$ converges unconditionally in $S^{\prime}\left(\mathbb{R}^{n}\right)$ for all admitted coefficients. We will not stress this point later on.

2.3.2. Wavelet frames: the case $s<0$. Let $k$ and $k^{\beta}$ be the non-negative functions introduced in (2.8)-(2.10) and used in Theorem 1. Let

$$
k^{\beta}(t, f)(x)=\int_{\mathbb{R}^{n}} k^{\beta}(y) f(x+t y) d y, \quad t>0, x \in \mathbb{R}^{n},
$$

be the corresponding local means for $f \in S^{\prime}\left(\mathbb{R}^{n}\right)$ (interpreted in the distributional sense). We use the abbreviation (2.12) now with $\varrho=0$ and put $\left\|\lambda\left|\ell_{p}\left\|_{s}=\right\| \lambda\right| \ell_{p}\right\|_{0, s}$. Let

$$
\left\|k(f) \mid \ell_{p}\right\|_{s}=\left(\sum_{\beta \in \mathbb{N}_{0}^{n}} \sum_{j=0}^{\infty} \sum_{m \in \mathbb{Z}^{n}} 2^{j(s-n / p) p}\left|k^{\beta}\left(2^{-j+J}, f\right)\left(2^{-j} m\right)\right|^{p}\right)^{1 / p}
$$

with the usual modification if $p=\infty$. The wavelets $\Phi^{\beta}\left(2^{j} x-m\right)$ have the same meaning as in (2.23) and (2.32).

Theorem 2. Let $1<p \leq \infty, s<0$.

(i) $f \in S^{\prime}\left(\mathbb{R}^{n}\right)$ is an element of $B_{p}^{s}\left(\mathbb{R}^{n}\right)$ if, and only if, it can be represented as

$$
f=\sum_{\beta, j, m} \lambda_{j m}^{\beta} \Phi^{\beta}\left(2^{j} x-m\right)
$$

with $\left\|\lambda \mid \ell_{p}\right\|_{s}<\infty$ and unconditional convergence in $S^{\prime}\left(\mathbb{R}^{n}\right)$. Furthermore,

$$
\left\|f\left|B_{p}^{s}\left(\mathbb{R}^{n}\right)\|\sim \inf \| \lambda\right| \ell_{p}\right\|_{s}
$$

where the infimum is taken over all admissible representations (2.36).

(ii) Any $f \in \mathcal{C}^{-\infty}\left(\mathbb{R}^{n}\right)$ can be represented as

$$
f=2^{J n} \sum_{\beta, j, m} k^{\beta}\left(2^{-j+J}, f\right)\left(2^{-j} m\right) \Phi^{\beta}\left(2^{j} x-m\right)
$$

with unconditional convergence in $S^{\prime}\left(\mathbb{R}^{n}\right)$. In addition, $f \in B_{p}^{s}\left(\mathbb{R}^{n}\right)$ if, and only if, $\left\|k(f) \mid \ell_{p}\right\|_{s}<\infty$.

(iii) Let $f \in B_{p}^{s}\left(\mathbb{R}^{n}\right)$. Then (2.37) is an optimal representation, in the sense that

$$
\left\|f\left|B_{p}^{s}\left(\mathbb{R}^{n}\right)\|\sim\| k(f)\right| \ell_{p}\right\|_{s}, \quad f \in B_{p}^{s}\left(\mathbb{R}^{n}\right),
$$

where the equivalence constants are independent of $f$. 
Remark 5. Part (iii) is not a surprise. Let $k(t, f)=k^{\beta}(t, f)$ if $\beta=0$ in (2.34). Then for some $r_{0}>0$ and all $r$ with $0<r \leq r_{0}$,

$$
\begin{aligned}
\left\|f \mid B_{p}^{s}\left(\mathbb{R}^{n}\right)\right\| & \sim\left(\sum_{j=0}^{\infty} 2^{j s p}\left\|k\left(2^{-j}, f\right) \mid L_{p}\left(\mathbb{R}^{n}\right)\right\|^{p}\right)^{1 / p} \\
& \sim\left(\sum_{j=0}^{\infty} \sum_{m \in \mathbb{Z}^{n}} 2^{j(s-n / p) p}\left|k\left(2^{-j}, f\right)\left(r 2^{-j} m\right)\right|^{p}\right)^{1 / p}
\end{aligned}
$$

The first assertion follows from equivalent norms in terms of local means, $[12,2.5 .3$, p. 138], and the additional observation that for $s<0$, one nonnegative kernel function $k(x)$ is sufficient. A discussion concerning this point may be found in $[14$, p. 125$]$, including references to the original papers. One can replace $k\left(2^{-j}, f\right)$ in the middle term of (2.39) by its maximal function $k^{*}\left(2^{-j}, f\right)_{a}$ according to $[12$, p. 134]. As a consequence one obtains the second equivalence in (2.39). An explicit formulation may also be found in $[17$, Theorem 4, pp. 16-17]. As above the influence of $\beta \in \mathbb{N}_{0}^{n}$ in (2.35), (2.38), is harmless. In analogy to (2.29) one can show that

$$
\left(\sum_{j=0}^{\infty} \sum_{m \in \mathbb{Z}^{n}} 2^{j(s-n / p) p}\left|k^{\beta}\left(2^{-j+J}, f\right)\left(2^{-j} m\right)\right|^{p}\right)^{1 / p} \leq c(\varrho) 2^{-\varrho|\beta|}\left\|f \mid B_{p}^{s}\left(\mathbb{R}^{n}\right)\right\|,
$$

where $c(\varrho)$ is independent of $\beta$. Hence (2.38) and (2.39) are equivalent assertions. In contrast to part (iii) one needs in the representation (2.37) the full sum over $\beta \in \mathbb{N}_{0}^{n}$ (at least in our approach).

2.3.3. Local regularity: the case $s<0$. If $f \in B_{p}^{s}\left(\mathbb{R}^{n}\right)$ with $1<p \leq \infty$ and $s<0$, then this does not say very much about the local regularity of $f$ in a ball or at a point. As indicated in the introduction, problems of this type attracted a lot of attention. We use the representation (2.37) for this purpose. If $f \in S^{\prime}\left(\mathbb{R}^{n}\right)$ and $g \in S^{\prime}\left(\mathbb{R}^{n}\right)$ then we write

$$
f=g \bmod C^{\infty} \quad \text { in } \Omega
$$

if the restriction of $f-g$ to the domain $\Omega$ is a $C^{\infty}$ function. Let $B(y, r)$ be the ball centred at $y \in \mathbb{R}^{n}$ and of radius $r>0$. Let $f$ be given by (2.37), $x^{0} \in \mathbb{R}^{n}$ and $K \in \mathbb{N}$. Then

$$
f^{K, x^{0}}=2^{J n} \sum_{\beta, j, m}^{K, x^{0}} k^{\beta}\left(2^{-j+J}, f\right)\left(2^{-j} m\right) \Phi^{\beta}\left(2^{j} x-m\right),
$$

where the summation is restricted to $j \in \mathbb{N}_{0}, m \in \mathbb{Z}^{n}$ with

$$
B\left(x^{0}, 2^{-K+1}\right) \cap B\left(2^{-j} m, 2^{-j+J}\right) \neq \emptyset .
$$

By (2.34) only the restriction of $f$ to $B\left(x^{0}, 2^{-K+2}\right)$ contributes to the terms with $j>K+J$ in (2.41). This results in a faithful description of the possibly improved local regularity of $f$ in a neighbourhood of $x^{0}$ and at the point $x^{0}$. 
Let

$$
\left\|k(f) \mid \ell_{p}\right\|_{s, K, x^{0}}=\left(\sum_{\beta, j, m}^{K, x^{0}} 2^{j(s-n / p) p}\left|k^{\beta}\left(2^{-j+J}, f\right)\left(2^{-j} m\right)\right|^{p}\right)^{1 / p}
$$

be the local counterpart of (2.35).

Corollary 1. Let $1<p \leq \infty, s<0$ and $f \in B_{p}^{s}\left(\mathbb{R}^{n}\right)$. Let $f$ and $f^{K, x^{0}}$ be represented by (2.37) and (2.41), respectively, where $x^{0} \in \mathbb{R}^{n}$ and $K \in \mathbb{N}$. Then

$$
f=f^{K, x^{0}} \bmod C^{\infty} \quad \text { in } B\left(x^{0}, 2^{-K-1}\right) .
$$

Furthermore, let $s \leq \sigma<0$. Then

$$
\left\|k(f) \mid \ell_{p}\right\|_{\sigma, K, x^{0}}<\infty \quad \text { implies } \quad f^{K, x^{0}} \in B_{p}^{\sigma}\left(\mathbb{R}^{n}\right),
$$

and, conversely,

$$
f^{K, x^{0}} \in B_{p}^{\sigma}\left(\mathbb{R}^{n}\right) \quad \text { implies } \quad\left\|k(f) \mid \ell_{p}\right\|_{\sigma, K+3, x^{0}}<\infty .
$$

REMARK 6 (Local regularity). Since (2.41) is a universal molecular representation, for any $\sigma \in \mathbb{R}$ and any $p$ with $0<p \leq \infty$ we have

$$
\left\|f^{K, x^{0}}\left|B_{p}^{\sigma}\left(\mathbb{R}^{n}\right)\|\leq c\| k(f)\right| \ell_{p}\right\|_{\sigma, K, x^{0}}
$$

where $c>0$ is independent of $f$. In other words, if the right-hand side of (2.47) is finite then $f^{K, x^{0}} \in B_{p}^{\sigma}\left(\mathbb{R}^{n}\right)$ and by (2.44),

$$
f \in B_{p}^{\sigma}\left(B\left(x^{0}, 2^{-K-1}\right)\right)
$$

Here $B_{p}^{\sigma}(\Omega)$ is the restriction of $B_{p}^{\sigma}\left(\mathbb{R}^{n}\right)$ to the domain $\Omega$, in our case the ball $B\left(x^{0}, 2^{-K-1}\right)$. If, in addition, $1<p \leq \infty$ and $\sigma<0$, then one gets not only a sufficient criterion but (almost) a characterization according to (2.45), (2.46). But (2.46) is no longer ensured in other cases. Just on the contrary. If, say, $1<p \leq \infty$, and $\sigma>0$, then the local means (2.34) with the non-negative kernels $k^{\beta}$ are no longer adequate. They must be replaced by the oscillating kernels $(-\Delta)^{L} k^{\beta}$ with $2 L>\sigma$. This will be done in Subsection 2.4 and has already been indicated in (1.4).

\subsection{Wavelet frames for functions}

2.4.1. L-wavelets. On the one hand, (2.37) is a universal representation for any $f \in \mathcal{C}^{-\infty}\left(\mathbb{R}^{n}\right)$. On the other hand, for given $p$ with $1<p \leq \infty$, satisfactory global and local regularity assertions as described in Theorem 2 and Corollary 1 are restricted to $s<0$. In principle it is clear how to circumvent this difficulty: Let $s \geq 0$ and $L \in \mathbb{N}$ be such that $s-2 L<0$. Then

$$
D_{L}=\mathrm{id}+(-\Delta)^{L}, \quad \text { where } \quad \Delta=\sum_{j=1}^{n} \frac{\partial^{2}}{\partial x_{j}^{2}}
$$


maps any space $B_{p}^{\sigma}\left(\mathbb{R}^{n}\right)$ with $\sigma \in \mathbb{R}, 0<p \leq \infty$, isomorphically onto $B_{p}^{\sigma-2 L}\left(\mathbb{R}^{n}\right)$. In particular, if $f \in B_{p}^{s}\left(\mathbb{R}^{n}\right)$ then one can apply the above representation, global and local regularity theory to $D_{L} f \in B_{p}^{s-2 L}\left(\mathbb{R}^{n}\right)$ and return afterwards via the inverse $D_{L}^{-1}$ of $D_{L}$ to $B_{p}^{s}\left(\mathbb{R}^{n}\right)$. By $(2.37)$ we have

$$
f=2^{J n} \sum_{\beta, j, m} k^{\beta}\left(2^{-j+J}, D_{L} f\right)\left(2^{-j} m\right) D_{L}^{-1}\left[\Phi^{\beta}\left(2^{j} \cdot-m\right)\right](x) .
$$

This is satisfactory as far as the coefficients are concerned: they remain local. The situation is not so clear for the lifted wavelets $\Phi^{\beta}\left(2^{j} x-m\right)$. However it turns out that the desired wavelet structure of $D_{L}^{-1}\left[\Phi^{\beta}\left(2^{j} \cdot-m\right)\right](x)$ is essentially preserved, at least as far as main terms are concerned. We introduce the resulting (father and mother) wavelets.

Definition 3. Let $\varphi_{0}, \varphi$, and $\Omega^{\beta}$ be given by (2.2), (2.3), and (2.16), respectively. Let $\beta \in \mathbb{N}_{0}^{n}$ and $L \in \mathbb{N}_{0}$. Then the father L-wavelets $\Phi_{\mathrm{F}}^{\beta, L}(x)$, the mother L-wavelets $\Phi_{\mathrm{M}}^{\beta, L}(x)$ and the remainder L-wavelets $\Phi_{j}^{\beta, L}(x)$ are given by

$$
\begin{aligned}
\left(\Phi_{\mathrm{F}}^{\beta, L}\right)^{\vee}(\xi) & =\frac{\varphi_{0}(\xi)}{1+|\xi|^{2 L}} \Omega^{\beta}(\xi), \quad \xi \in \mathbb{R}^{n}, \\
\left(\Phi_{\mathrm{M}}^{\beta, L}\right)^{\vee}(\xi) & =\frac{\varphi(\xi)}{|\xi|^{2 L}} \Omega^{\beta}(\xi), \quad \xi \in \mathbb{R}^{n}, \\
\left(\Phi_{j}^{\beta, L}\right)^{\vee}(\xi) & =-\frac{\varphi(\xi)}{|\xi|^{2 L}\left(|\xi|^{2 L}+2^{-2 L j}\right)} \Omega^{\beta}(\xi), \quad \xi \in \mathbb{R}^{n},
\end{aligned}
$$

if $j \in \mathbb{N}$ and $\Phi_{j}^{\beta, L}(\xi)=0$ if $j=0$.

REMARK 7. All these wavelets are elements of $S\left(\mathbb{R}^{n}\right)$. If $L=0$ then we obtain essentially the father wavelets and mother wavelets introduced in Definition 2,

$$
\Phi_{\mathrm{F}}^{\beta}=2 \Phi_{\mathrm{F}}^{\beta, 0} \quad \text { and } \quad \Phi_{\mathrm{M}}^{\beta}=\Phi_{\mathrm{M}}^{\beta, 0}=-2 \Phi_{j}^{\beta, 0} \quad \text { for } j \in \mathbb{N}, \beta \in \mathbb{N}_{0}^{n} .
$$

In particular, by (2.20) we have the counterpart of (2.19) with $\Phi_{\mathrm{M}}^{\beta, L}$ and $\Phi_{j}^{\beta, L}$ in place of $\Phi_{\mathrm{M}}^{\beta}$, and also corresponding counterparts of (2.21), (2.22). We generalize (2.23) by setting

$$
\Phi^{\beta, L}\left(2^{j} x-m\right)= \begin{cases}\Phi_{\mathrm{F}}^{\beta, L}(x-m) & \text { if } j=0, \\ \Phi_{\mathrm{M}}^{\beta, L}\left(2^{j} x-m\right) & \text { if } j \in \mathbb{N} .\end{cases}
$$

The suitable counterparts of (2.31), (2.32) will be given by

$$
f=\sum_{\beta, j, m} \lambda_{j m}^{\beta}\left(\Phi^{\beta, L}+2^{-2 j L} \Phi_{j}^{\beta, L}\right)\left(2^{j} x-m\right)
$$


and

$$
\left\{2^{-j(s-n / p)}\left(\Phi^{\beta, L}+2^{-2 j L} \Phi_{j}^{\beta, L}\right)\left(2^{j} x-m\right): j \in \mathbb{N}_{0}, m \in \mathbb{Z}^{n}\right\}, \beta \in \mathbb{N}_{0}^{n} .
$$

As with (2.31), (2.32), it follows by the above remarks and again by [2] that for fixed $\beta \in \mathbb{N}_{0}^{n}$ the functions in (2.56) are normalized molecules in $B_{p}^{s}\left(\mathbb{R}^{n}\right)$. The remainder $L$-wavelets even generate

$$
\left\{2^{-j(s-n / p)-2 j L} \Phi_{v}^{\beta, L}\left(2^{j} x-m\right): j \in \mathbb{N}_{0}, m \in \mathbb{Z}^{n}\right\}, \quad v \in \mathbb{N}, \beta \in \mathbb{N}_{0}^{n},
$$

normalized molecules in $B_{p}^{s+2 L}\left(\mathbb{R}^{n}\right)$, uniformly with respect to $v \in \mathbb{N}$. Here we need only $v=j$. As in the case of $L=0$ the influence of $\beta \in \mathbb{N}_{0}^{n}$ is harmless. As before one always has estimates of type (2.29), where in the case of optimal coefficients in (2.55) the number $\varrho \geq 0$ can be prescribed. In other words, compared with the case $L=0$ considered in 2.3 there is now only one new point: If $L \in \mathbb{N}$, then we have in addition the remainder $L$-wavelets $\Phi_{j}^{\beta, L}$. But the related terms have regularity $s+2 L$, compared to the expected regularity $s$ of the main terms. In addition, $L \in \mathbb{N}$ can be chosen arbitrarily large.

2.4.2. Wavelet frames: the case $s>0$. Let $1<p \leq \infty$. The main assertions in 2.3.2 and 2.3.3 are restricted to the case $B_{p}^{s}\left(\mathbb{R}^{n}\right)$ with $s<0$. Using 2.4.1 one can extend this theory to $s \in \mathbb{R}$. But since the results for $s<0$ obtained so far are satisfactory it seems to be reasonable to restrict our attention now to $s>0$ from the very beginning and to assume $f \in L_{p}\left(\mathbb{R}^{n}\right)$ to start from. First we generalize the local means in (2.34). Let $k$ and $k^{\beta}$ be the non-negative functions introduced in (2.8)-(2.10), complemented now by

$$
k_{L}^{\beta}(x)=(-\Delta)^{L} k^{\beta}(x), \quad L \in \mathbb{N}_{0}, x \in \mathbb{R}^{n},
$$

where $\Delta$ is the Laplacian as in (2.48). Let

$$
k_{L}^{\beta}(t, f)(x)=\int_{\mathbb{R}^{n}} k_{L}^{\beta}(y) f(x+t y) d y, \quad t>0, x \in \mathbb{R}^{n},
$$

be the corresponding local means for $f \in S^{\prime}\left(\mathbb{R}^{n}\right)$ (interpreted in the distributional sense). If $L=0$, then we have $k_{0}^{\beta}(t, f)=k^{\beta}(t, f)$ according to (2.34). Again we use the abbreviation (2.12) now with $\varrho=0$ and put

$$
\left\|\lambda\left|\ell_{p}\left\|_{s}=\right\| \lambda\right| \ell_{p}\right\|_{0, s}
$$

Let $\left\|k_{L}(f) \mid \ell_{p}\right\|_{s}$ be given by (2.35) with $k_{L}^{\beta}$ in place of $k^{\beta}$. The wavelets $\Phi^{\beta, L}\left(2^{j} x-m\right)$ and $\Phi_{j}^{\beta, L}\left(2^{j} x-m\right)$ have the same meaning as in $(2.50)-(2.52)$ and (2.54).

TheOREM 3. Let $1<p \leq \infty, L \in \mathbb{N}$, and $0<s<2 L$.

(i) $f \in L_{p}\left(\mathbb{R}^{n}\right)$ is an element of $B_{p}^{s}\left(\mathbb{R}^{n}\right)$ if, and only if, it can be represented as 


$$
f=\sum_{\beta, j, m} \lambda_{j m}^{\beta}\left(\Phi^{\beta, L}+2^{-2 j L} \Phi_{j}^{\beta, L}\right)\left(2^{j} x-m\right)
$$

with $\left\|\lambda \mid \ell_{p}\right\|_{s}<\infty$ and absolute, and hence unconditional, convergence in $L_{p}\left(\mathbb{R}^{n}\right)$. Furthermore,

$$
\left\|f\left|B_{p}^{s}\left(\mathbb{R}^{n}\right)\|\sim \inf \| \lambda\right| \ell_{p}\right\|_{s}
$$

where the infimum is taken over all admissible representations (2.58).

(ii) Any $f \in B_{p}^{s}\left(\mathbb{R}^{n}\right)$ can be represented as

$$
f=2^{J(n-2 L)} \sum_{\beta, j, m} \lambda_{j m}^{\beta}(f)\left(\Phi^{\beta, L}+2^{-2 j L} \Phi_{j}^{\beta, L}\right)\left(2^{j} x-m\right)
$$

with

$$
\lambda_{j m}^{\beta}(f)=\left[k_{L}^{\beta}\left(2^{-j+J}, f\right)+2^{-2(j-J) L} k^{\beta}\left(2^{-j+J}, f\right)\right]\left(2^{-j} m\right)
$$

and absolute convergence in $L_{p}\left(\mathbb{R}^{n}\right)$. This representation is optimal in the sense that

$$
\left\|f\left|B_{p}^{s}\left(\mathbb{R}^{n}\right)\|\sim\| \lambda(f)\right| \ell_{p}\right\|_{s} \sim\left\|k_{L}(f)\left|\ell_{p}\left\|_{s}+\right\| f\right| L_{p}\left(\mathbb{R}^{n}\right)\right\|,
$$

where the equivalence constants are independent of $f$.

REMARK 8. The assumptions $f \in L_{p}\left(\mathbb{R}^{n}\right)$ and $s>0$ are convenient for us. They are justified by Subsection 2.3. But they are not necessary. For example one may assume $f \in \mathcal{C}^{-\infty}\left(\mathbb{R}^{n}\right)$. Then one gets an extension of Theorem 2(ii), where $f$ is now represented by $(2.60),(2.61)$ with $L \in \mathbb{N}_{0}$. In particular, if $L=0$, then by (2.53) this representation coincides with (2.37) (as it should be). Furthermore we refer again to the equivalent norms in $B_{p}^{s}\left(\mathbb{R}^{n}\right)$ in terms of local means, $[12,2.5 .3$, p. 138], which cover essentially the equivalences in (2.62). A more detailed discussion of this point has been given in Remark 5 which also applies to the above situation.

2.4.3. Global and local regularity: the case $s>0$. Let $\Omega$ be a domain in $\mathbb{R}^{n}$. If $f \in S^{\prime}\left(\mathbb{R}^{n}\right)$ and $g \in S^{\prime}\left(\mathbb{R}^{n}\right)$ then we write, in analogy to (2.40),

$$
f=g \bmod B_{p}^{\sigma} \quad \text { in } \Omega
$$

if the restriction of $f-g$ to $\Omega$ belongs to $B_{p}^{\sigma}(\Omega)$. First we look at the global representations (2.60), (2.61). Since $f \in L_{p}\left(\mathbb{R}^{n}\right)$ we have $f \in B_{p}^{-\varepsilon}\left(\mathbb{R}^{n}\right)$ for any $\varepsilon>0$. Let temporarily

$$
\lambda_{j m}^{\prime \beta}(f)=2^{-2 j L} k^{\beta}\left(2^{-j+J}, f\right)\left(2^{-j} m\right) .
$$

Then by Theorem 2(iii) we obtain, in obvious notation,

$$
\left\|\lambda^{\prime}(f)\left|\ell_{p}\left\|_{2 L-\varepsilon}=\right\| k(f)\right| \ell_{p}\right\|_{-\varepsilon} \sim\left\|f \mid B_{p}^{-\varepsilon}\left(\mathbb{R}^{n}\right)\right\| .
$$

Let $f^{\prime}$ be given by (2.60) with $\lambda^{\prime}$ in place of $\lambda$. Since (2.60) is a univer- 
sal molecular representation we get, by modifying the proof in Step 2 of Subsection 3.1 below (based as there on (2.15) with $\beta$ ! in the denominator),

$$
f^{\prime} \in B_{p}^{2 L-\varepsilon}\left(\mathbb{R}^{n}\right), \quad\left\|f^{\prime}\left|B_{p}^{2 L-\varepsilon}\left(\mathbb{R}^{n}\right)\|\leq c\| \lambda^{\prime}(f)\right| \ell_{p}\right\|_{2 L-\varepsilon} .
$$

Let

$$
f^{\prime \prime}=\sum_{\beta, j, m} 2^{-2 j L} k_{L}^{\beta}\left(2^{-j+J}, f\right)\left(2^{-j} m\right) \Phi_{j}^{\beta, L}\left(2^{j} x-m\right) .
$$

Then by (2.62) and the same arguments as in (2.63) we obtain

$$
f^{\prime \prime} \in B_{p}^{s+2 L}\left(\mathbb{R}^{n}\right), \quad\left\|f^{\prime \prime}\left|B_{p}^{s+2 L}\left(\mathbb{R}^{n}\right)\|\leq c\| f\right| B_{p}^{s}\left(\mathbb{R}^{n}\right)\right\| .
$$

In other words, by (2.60) we have, for any $\varepsilon>0$,

$$
f=2^{J(n-2 L)} \sum_{\beta, j, m} k_{L}^{\beta}\left(2^{-j+J}, f\right)\left(2^{-j} m\right) \Phi^{\beta, L}\left(2^{j} x-m\right) \bmod B_{p}^{2 L-\varepsilon}
$$

in $\mathbb{R}^{n}$. Hence the right-hand side of (2.64) is the main term of $f$ as far as global regularity is concerned. As for local regularity there is no problem to repeat the considerations from 2.3.3. Let $x^{0} \in \mathbb{R}^{n}, K \in \mathbb{N}$ and let as there

$$
f_{L}^{K, x^{0}}=2^{J(n-2 L)} \sum_{\beta, j, m}^{K, x^{0}} \lambda_{j m}^{\beta}(f)\left(\Phi^{\beta, L}+2^{-2 j L} \Phi_{j}^{\beta, L}\right)\left(2^{j} x-m\right)
$$

with $(2.61)$ and

$$
\widetilde{f}_{L}^{K, x^{0}}=2^{J(n-2 L)} \sum_{\beta, j, m}^{K, x^{0}} k_{L}^{\beta}\left(2^{-j+J}, f\right)\left(2^{-j} m\right) \Phi^{\beta, L}\left(2^{j} x-m\right),
$$

where the summation is restricted to $j \in \mathbb{N}_{0}, m \in \mathbb{Z}^{n}$, with (2.42). Let $\left\|k_{L}(f) \mid \ell_{p}\right\|_{s, K, x^{0}}$ be given by (2.43) with $k_{L}^{\beta}$ in place of $k^{\beta}$.

Corollary 2. Let $1<p \leq \infty, L \in \mathbb{N}$, and $0<s<2 L$. Let $f \in B_{p}^{s}\left(\mathbb{R}^{n}\right)$, $f_{L}^{K, x^{0}}$, and $\widetilde{f}_{L}^{K, x^{0}}$ be represented by (2.60) with (2.61), (2.65), and (2.66), respectively, where $x^{0} \in \mathbb{R}^{n}$ and $K \in \mathbb{N}$. Then

$$
f=f_{L}^{K, x^{0}} \bmod C^{\infty} \quad \text { in } B\left(x^{0}, 2^{-K-1}\right)
$$

and for any $\varepsilon>0$,

$$
f=\widetilde{f}_{L}^{K, x^{0}} \bmod B_{p}^{2 L-\varepsilon} \quad \text { in } B\left(x^{0}, 2^{-K-1}\right) .
$$

Furthermore, let $s \leq \sigma<2 L$. Then

$$
\left\|k_{L}(f) \mid \ell_{p}\right\|_{\sigma, K, x^{0}}<\infty \quad \text { implies } \quad \widetilde{f}_{L}^{K, x^{0}} \in B_{p}^{\sigma}\left(\mathbb{R}^{n}\right)
$$

and, conversely,

$$
\widetilde{f}_{L}^{K, x^{0}} \in B_{p}^{\sigma}\left(\mathbb{R}^{n}\right) \quad \text { implies } \quad\left\|k_{L}(f) \mid \ell_{p}\right\|_{\sigma, K+3, x^{0}}<\infty .
$$

Remark 9. This is the counterpart of Corollary 1. Remark 6 can be carried over. 


\subsection{Pointwise regularity}

2.5.1. Some preparations. So far we have, by Corollaries 1 and 2, satisfactory assertions concerning the local regularity of distributions and functions in balls $B\left(x^{0}, 2^{-K}\right)$. It is of interest what happens if $K \rightarrow \infty$. It is just this question which attracted a lot of attention in some of the papers mentioned in the Introduction. If $f \in S^{\prime}\left(\mathbb{R}^{n}\right)$ then sing supp $f$ has the usual meaning: the collection of all points $y \in \mathbb{R}^{n}$ such that there is no domain $\Omega$ with $y \in \Omega$ in which $f$ is $C^{\infty}$.

Definition 4. Let $1<p \leq \infty, f \in B_{p}^{-\infty}\left(\mathbb{R}^{n}\right)$ and $x^{0} \in \operatorname{sing} \operatorname{supp} f$. Let $t=1 / p$. Then

$s_{f}\left(x^{0}, t\right)=\sup \left\{\sigma \in \mathbb{R}:\right.$ there is a $K \in \mathbb{N}$ with $\left.f \in B_{p}^{\sigma}\left(B\left(x^{0}, 2^{-K}\right)\right)\right\}$.

REMARK 10. By the discussion at the beginning of 2.3.1 this definition applies in particular to any compactly supported distribution $f \in D^{\prime}\left(\mathbb{R}^{n}\right)$. By (2.30), we have

$$
-\infty<s_{f}\left(x^{0}, t\right) \leq \infty
$$

where it might happen that $s_{f}\left(x^{0}, t\right)=\infty$ at some points $x^{0} \in \operatorname{sing} \operatorname{supp} f$. Of course, if $f \in B_{p}^{\sigma}\left(B\left(x^{0}, 2^{-K}\right)\right)$ then $f \in B_{p}^{\sigma}\left(B\left(x^{0}, 2^{-K^{\prime}}\right)\right)$ for any $K^{\prime} \in \mathbb{N}$ with $K^{\prime}>K$.

2.5.2. Pointwise regularity assertions. Let, as before Corollary 2,

$$
\left\|k_{L}(f) \mid \ell_{p}\right\|_{\sigma, K, x^{0}}=\left(\sum_{\beta, j, m}^{K, x^{0}} 2^{j(\sigma-n / p) p}\left|k_{L}^{\beta}\left(2^{-j+J}, f\right)\left(2^{-j} m\right)\right|^{p}\right)^{1 / p}
$$

with the usual modification if $p=\infty$.

Theorem 4. Let $1<p \leq \infty, f \in B_{p}^{-\infty}\left(\mathbb{R}^{n}\right)$ and $x^{0} \in \operatorname{sing} \operatorname{supp} f$. Let $t=1 / p$. Then $s_{f}\left(x^{0}, t\right)<\infty$ if, and only if, there is an $L \in \mathbb{N}_{0}$ such that

$$
\left\|k_{L}(f) \mid \ell_{p}\right\|_{2 L-\varepsilon, K, x^{0}}=\infty
$$

for some $\varepsilon>0$ and all $K \in \mathbb{N}$. Let $s_{f}\left(x^{0}, t\right)<\infty$ and let $L_{0}=L\left(f, x^{0}\right) \in \mathbb{N}_{0}$ be the smallest $L$ satisfying (2.72). Then

$$
s_{f}\left(x^{0}, t\right)=\sup \left\{s \in \mathbb{R}:\left\|k_{L_{0}}(f) \mid \ell_{p}\right\|_{s, K, x^{0}}<\infty \text { for some } K \in \mathbb{N}\right\} .
$$

REMARK 11. Of course, if $K^{\prime}>K$, then

$$
\left\|k_{L_{0}}(f) \mid \ell_{p}\right\|_{s, K^{\prime}, x^{0}}<\infty \text { if }\left\|k_{L_{0}}(f) \mid \ell_{p}\right\|_{s, K, x^{0}}<\infty .
$$

Hence of interest is the behaviour of $\left\|k_{L_{0}}(f) \mid \ell_{p}\right\|_{s, K, x^{0}}$ as $K \rightarrow \infty$. Since the above criterion is necessary and sufficient it characterizes also those points $x^{0} \in \operatorname{sing} \operatorname{supp} f$ where $s_{f}\left(x^{0}, t\right)=\infty$. To get a better understanding of (2.73) we add a discussion. First let $L_{0}=L\left(f, x^{0}\right)=0$. Then both by Corollary 1 and by (2.73),

$$
s_{f}\left(x^{0}, t\right) \leq-\varepsilon<0 .
$$


Let $L_{0} \in \mathbb{N}$. Then the right-hand inequality of

$$
2\left(L_{0}-1\right) \leq s_{f}\left(x^{0}, t\right)<2 L_{0}
$$

is a consequence of Corollary 2. The left-hand inequality follows from the assumption that $L_{0} \in \mathbb{N}$ is the smallest number with (2.72) and again Corollary 2.

REMARK 12. The above theorem gives a satisfactory characterization of pointwise regularity for distributions in terms of the local means $k_{L}^{\beta}(t, f)$ introduced in (2.57) and of the norms in (2.71). From the point of view of wavelet theory one may ask whether the summation over $\beta \in \mathbb{N}_{0}^{n}$ is really necessary. This might be the case (in our approach) as far as representations of distributions and functions are concerned as described in Theorems 2 and 3. But for criteria of pointwise regularity of distributions the situation is different. We describe a corresponding result which complements (2.45) and (2.69) in Corollaries 1 and 2, respectively. This can be used afterwards to modify some assertions of the above theorem. But this will not be done here in detail. First we introduce some notation. We fix $J \in \mathbb{N}$ as in (2.9). Let now $k$ be a compactly supported $C^{\infty}$ function in $\mathbb{R}^{n}$ and again let $k_{L}(x)=(-\Delta)^{L} k(x)$, where $L \in \mathbb{N}_{0}$. Let

$$
k_{L}(t, f)(x)=\int_{\mathbb{R}^{n}} k_{L}(y) f(x+t y) d y, \quad t>0, x \in \mathbb{R}^{n},
$$

be the local means introduced in (2.57) with $\beta=0$. Modifying (2.71), let

$$
\left\|k_{L}(f) \mid \ell_{p}\right\|_{\sigma, K, x^{0}}^{0}=\left(\sum_{j, m}^{K, x^{0}} 2^{j(\sigma-n / p) p}\left|k_{L}\left(2^{-j+J}, f\right)\left(2^{-j} m\right)\right|^{p}\right)^{1 / p}
$$

and

$$
\left\|k_{L}(f)\left|\ell_{p}\left\|_{\sigma, K, x^{0}}^{\sup }=\sup \right\| k_{L}(f)\right| \ell_{p}\right\|_{\sigma, K, x^{0}}^{0}
$$

where the supremum is taken over all $C^{\infty}$ functions $k$ with

$$
\operatorname{supp} k \subset\{y:|y|<1\}, \quad\left|D^{\alpha} k(x)\right| \leq 1 \quad \text { if }|\alpha| \leq 2 L \text { and } x \in \mathbb{R}^{n} .
$$

Otherwise we use the notation as before, hence $L \in \mathbb{N}_{0}, \sigma \in \mathbb{R}, 1 \leq p \leq \infty$ (with the usual modification if $p=\infty$ in (2.76)); and the summation over $j \in \mathbb{N}_{0}$ and $m \in \mathbb{Z}^{n}$ in (2.76) is restricted to those couples $(j, m)$ with (2.42). If $L=0$ then we write $k$ in place of $k_{0}$ in (2.75)-(2.77).

Proposition 2. Let $1<p \leq \infty$. Let $x^{0} \in \mathbb{R}^{n}$ and $K \in \mathbb{N}$.

(i) Let $s \leq \sigma<0$ and $f \in B_{p}^{s}\left(\mathbb{R}^{n}\right)$. Let $f$ and $f^{K, x^{0}}$ be represented by (2.37) and (2.41), respectively. Then

$$
\left\|k(f) \mid \ell_{p}\right\|_{\sigma, K, x^{0}}^{\text {sup }}<\infty \quad \text { implies } \quad f^{K, x^{0}} \in B_{p}^{\sigma}\left(\mathbb{R}^{n}\right) .
$$


(ii) Let $L \in \mathbb{N}, 0<s \leq \sigma<2 L$ and $f \in B_{p}^{s}\left(\mathbb{R}^{n}\right)$. Let $f$ and $\widetilde{f} K, x^{0}$ be represented by (2.60) and (2.66), respectively. Then

$$
\left\|k_{L}(f) \mid \ell_{p}\right\|_{\sigma, K, x^{0}}^{\sup }<\infty \quad \text { implies } \quad \tilde{f}^{K, x^{0}} \in B_{p}^{\sigma}\left(\mathbb{R}^{n}\right) .
$$

REMARK 13. This proposition complements Corollaries 1 and 2. Instead of the summation over $\beta \in \mathbb{N}_{0}^{n}$ we now have the supremum in (2.77). But this is a rather typical situation in connection with local means. By (2.44) and (2.68) one gets from (2.79) and (2.80) assertions about improved local regularity of $f$. This can be extended to assertions of pointwise regularity according to Theorem 4. Furthermore there is a converse to (2.79), (2.80). Let $s \in \mathbb{R}, L \in \mathbb{N}_{0}$ with $2 L>s$ and $1<p \leq \infty$. Let $k$ be a $C^{\infty}$ function with compact support. Then there is a positive number $c$ such that

$$
\left(\sum_{j=0}^{\infty} \sum_{m \in \mathbb{Z}^{n}} 2^{j(s-n / p) p}\left|k_{L}\left(2^{-j+J}, f\right)\left(2^{-j} m\right)\right|^{p}\right)^{1 / p} \leq c\left\|f \mid B_{p}^{s}\left(\mathbb{R}^{n}\right)\right\|
$$

for all $f \in B_{p}^{s}\left(\mathbb{R}^{n}\right)$. This is covered by the references mentioned in Remark 5. Based on this observation both parts of this proposition can be complemented as follows: We use the norms in (2.76), again with $k$ in place of $k_{L}$ if $L=0$. Let $K \in \mathbb{N}$ and let $1<p \leq \infty$.

(i) Let $s \leq \sigma<0$. Then

$$
f^{K, x^{0}} \in B_{p}^{\sigma}\left(\mathbb{R}^{n}\right) \quad \text { implies }\left\|k(f) \mid \ell_{p}\right\|_{\sigma, K+3, x^{0}}^{0}<\infty .
$$

(ii) Let $L \in \mathbb{N}$ and $0<s \leq \sigma<2 L$. Then

$$
\tilde{f}^{K, x^{0}} \in B_{p}^{\sigma}\left(\mathbb{R}^{n}\right) \quad \text { implies } \quad\left\|k_{L}(f) \mid \ell_{p}\right\|_{\sigma, K+3, x^{0}}^{0}<\infty .
$$

These are the counterparts of (2.46) and (2.70) without the additional assumption (2.9), and obviously restricted to the terms with $\beta=0$.

\section{PROOFS}

\subsection{Proof of Theorem 1}

Step 1. First we assume that $f$ is given by (2.25) with $\left\|\lambda \mid \ell_{p}\right\|_{\varrho, s}<\infty$ for some $\varrho \geq 0$. Let

$$
f=\sum_{\beta} f^{\beta} \quad \text { with } \quad f^{\beta}=\sum_{j, m} \lambda_{j m}^{\beta} k^{\beta}\left(2^{j-J} x-2^{-J} m\right) .
$$

We use atomic decompositions for the spaces $B_{p}^{s}\left(\mathbb{R}^{n}\right)$ with $s>0$ and $1 \leq$ $p \leq \infty$ as described in [13, Theorem 13.8, p. 75]. By (2.8) the support of $k$ is also contained in an open ball centred at the origin and of radius $2^{-\varepsilon}$ for some $\varepsilon>0$. Then it follows easily that

$$
\left\{2^{\varepsilon|\beta|} 2^{-j(s-n / p)} k^{\beta}\left(2^{j-J} x-2^{-J} m\right): j \in \mathbb{N}_{0}, m \in \mathbb{Z}^{n}\right\}, \quad \beta \in \mathbb{N}_{0}^{n},
$$


are admitted systems of atoms, provided we ignore constants which are independent of $j, m, \beta$. Hence $f^{\beta} \in B_{p}^{s}\left(\mathbb{R}^{n}\right)$ and

$$
\left\|f^{\beta} \mid B_{p}^{s}\left(\mathbb{R}^{n}\right)\right\| \leq c 2^{-\varepsilon|\beta|}\left(\sum_{j=0}^{\infty} \sum_{m \in \mathbb{Z}^{n}} 2^{j(s-n / p) p}\left|\lambda_{j m}^{\beta}\right|^{p}\right)^{1 / p},
$$

where $c$ is independent of $\beta$. Summation over $\beta$ proves $f \in B_{p}^{s}\left(\mathbb{R}^{n}\right)$ and

$$
\left\|f\left|B_{p}^{s}\left(\mathbb{R}^{n}\right)\|\leq c\| \lambda\right| \ell_{p}\right\|_{\varrho, s}
$$

for any $\varrho \geq 0$. As for the absolute convergence of the series in (3.1) in $L_{p}\left(\mathbb{R}^{n}\right)$ we refer to $[14,1.4$, pp. $7-9$, and 2.7 , p. 14], where we studied this question in detail.

Step 2. We construct optimal coefficients according to (2.24), (2.27), (2.28). Then one gets as a by-product the converse to (3.3), which proves part (i). For this purpose we adapt the relevant arguments in [14, pp. 17-24], to our situation. Let $f \in B_{p}^{s}\left(\mathbb{R}^{n}\right)$ with $1 \leq p \leq \infty$ and $s>0$. Let $Q_{j}$ with $j \in \mathbb{N}_{0}$ be cubes in $\mathbb{R}^{n}$ centred at the origin and with side-length $2 \pi 2^{j}$. In particular, $\operatorname{supp} \varphi_{j} \subset Q_{j}$, where $\varphi_{j}$ are given by (2.2), (2.3) and we have

$$
\widehat{f}(\xi)=\sum_{j=0}^{\infty} \varphi_{j}(\xi) \widehat{f}(\xi), \quad \xi \in \mathbb{R}^{n} .
$$

We expand $\varphi_{j} \widehat{f}$ in $Q_{j}$ in its trigonometric series and obtain

$$
\left(\varphi_{j} \widehat{f}\right)(\xi)=\sum_{m \in \mathbb{Z}^{n}} b_{j m} \exp \left(-i 2^{-j} m \xi\right), \quad \xi \in Q_{j},
$$

with

$$
\begin{aligned}
b_{j m} & =(2 \pi)^{-n} 2^{-j n} \int_{Q_{j}}\left(\varphi_{j} \widehat{f}\right)(\xi) \exp \left(i 2^{-j} m \xi\right) d \xi \\
& =(2 \pi)^{-n / 2} 2^{-j n}\left(\varphi_{j} \widehat{f}\right)^{\vee}\left(2^{-j} m\right) .
\end{aligned}
$$

By Proposition 1 we have

$$
\left\|f \mid B_{p}^{s}\left(\mathbb{R}^{n}\right)\right\| \sim\left(\sum_{j=0}^{\infty} 2^{j s p} 2^{j n(1-1 / p) p}\left|b_{j m}\right|^{p}\right)^{1 / p}, \quad 1 \leq p \leq \infty
$$

(with the usual modification if $p=\infty$ ). Let $\omega$ be given by (2.14) and let $\omega_{j}(x)=\omega\left(2^{-j} x\right)$. Then $\omega_{j}$ has a compact support in $Q_{j}$ and it follows by (3.4) that

$$
\begin{aligned}
\left(\varphi_{j} \widehat{f}\right)^{\vee}(x) & =\sum_{m \in \mathbb{Z}^{n}} b_{j m} \omega_{j}^{\vee}\left(x-2^{-j} m\right) \\
& =2^{j n} \sum_{m \in \mathbb{Z}^{n}} b_{j m} \omega^{\vee}\left(2^{j} x-m\right), \quad x \in \mathbb{R}^{n} .
\end{aligned}
$$


Let $k$ be given by (2.8), (2.9). Expanding the analytic function $\omega^{\vee}\left(2^{j} x-m\right)$ at $2^{-j} l$ with $l \in \mathbb{Z}^{n}$ we get

$$
\begin{aligned}
k\left(2^{j-J} x\right. & \left.-2^{-J} l\right) \omega^{\vee}\left(2^{j} x-m\right) \\
& =\sum_{\beta \in \mathbb{N}_{0}^{n}} \frac{2^{j|\beta|}}{\beta !}\left(D^{\beta} \omega^{\vee}\right)(l-m)\left(x-2^{-j} l\right)^{\beta} k\left(2^{j-J} x-2^{-J} l\right) \\
& =\sum_{\beta \in \mathbb{N}_{0}^{n}} \frac{2^{J|\beta|}}{\beta !}\left(D^{\beta} \omega^{\vee}\right)(l-m) k^{\beta}\left(2^{j-J} x-2^{-J} l\right),
\end{aligned}
$$

where we used (2.10). By (2.9), (3.7), (3.8) we obtain

$$
\begin{aligned}
\left(\varphi_{j} \widehat{f}\right)^{\vee}(x) & =\sum_{m \in \mathbb{Z}^{n}} 2^{j n} b_{j m} \sum_{l \in \mathbb{Z}^{n}} k\left(2^{j-J} x-2^{-J} l\right) \omega^{\vee}\left(2^{j} x-m\right) \\
& =\sum_{\beta \in \mathbb{N}_{0}^{n}} \sum_{l \in \mathbb{Z}^{n}} k^{\beta}\left(2^{j-J} x-2^{-J} l\right) \sum_{m \in \mathbb{Z}^{n}} 2^{j n} b_{j m} \frac{2^{J|\beta|}}{\beta !}\left(D^{\beta} \omega^{\vee}\right)(l-m) .
\end{aligned}
$$

Hence,

$$
f=\sum_{j=0}^{\infty} \sum_{\beta \in \mathbb{N}_{0}^{n}} \sum_{l \in \mathbb{Z}^{n}} \lambda_{j l}^{\beta} k^{\beta}\left(2^{j-J} x-2^{-J} l\right)
$$

with

$$
\lambda_{j l}^{\beta}=\sum_{m \in \mathbb{Z}^{n}} 2^{j n} b_{j m} \frac{2^{J|\beta|}}{\beta !}\left(D^{\beta} \omega^{\vee}\right)(l-m) .
$$

First we check that $\lambda_{j l}^{\beta}$ are optimal coefficients. In other words, if $\varrho \geq 0$ is given then we must find a constant $c$ such that, according to (2.11), (2.12),

$$
\left\|\lambda\left|\ell_{p}\left\|_{\varrho, s} \leq c\right\| f\right| B_{p}^{s}\left(\mathbb{R}^{n}\right)\right\| \quad \text { for all } f \in B_{p}^{s}\left(\mathbb{R}^{n}\right) .
$$

In $[15,3.1 .1]$, we proved that for any given $a>0$ there are constants $C>0$ and $c_{a}>0$ such that

$$
\left|D^{\beta} \omega^{\vee}(x)\right| \leq c_{a} 2^{C|\beta|}\left(1+|x|^{2}\right)^{-a}, \quad x \in \mathbb{R}^{n}, \beta \in \mathbb{N}_{0}^{n},
$$

where $C$ is independent of $x, a, \beta$, and $c_{a}$ is independent of $x, \beta$. Interpreting (3.9) as a convolution in $\ell_{p}$ it follows that for any $\varrho \geq 0$ there is a constant $c(\varrho)$ such that

$$
\left(\sum_{l \in \mathbb{Z}^{n}}\left|\lambda_{j l}^{\beta}\right|^{p}\right)^{1 / p} \leq c(\varrho) 2^{-(\varrho+1)|\beta|}\left(\sum_{l \in \mathbb{Z}^{n}}\left|2^{j n} b_{j l}\right|^{p}\right)^{1 / p} .
$$

Now (3.10) follows from (3.6), (3.12). The proof of part (i) is complete.

Step 3. It remains to prove that the optimal coefficients $\lambda_{j l}^{\beta}$ in (3.9) can be represented by (2.24). By (3.5) and well-known properties of the Fourier 
transform we have

$$
2^{j n} b_{j m}=(2 \pi)^{-n} \int_{\mathbb{R}^{n}}\left(\varphi_{j}\right)^{\vee}\left(2^{-j} m-y\right) f(y) d y, \quad j \in \mathbb{N}_{0},
$$

and hence by $(2.3)$,

$$
2^{j n} b_{j m}=(2 \pi)^{-n} 2^{j n} \int_{\mathbb{R}^{n}} \varphi^{\vee}\left(m-2^{j} y\right) f(y) d y, \quad j \in \mathbb{N} .
$$

Recall $\left(D^{\beta} \omega^{\vee}\right)(\xi)=i^{|\beta|}\left(x^{\beta} \omega(x)\right)^{\vee}(\xi)$. Using (2.15) we obtain, for $j \in \mathbb{N}$,

$$
\lambda_{j l}^{\beta}=2^{j n} \int_{\mathbb{R}^{n}} f(y) \sum_{m \in \mathbb{Z}^{n}}\left(\omega^{\beta}\right)^{\vee}(l-m) \varphi^{\vee}\left(m-2^{j} y\right) d y .
$$

Replacing $l-m$ by $m$ and using $\varphi^{\vee}(z)=\widehat{\varphi}(-z)$ one gets

$$
\begin{aligned}
\lambda_{j l}^{\beta} & =2^{j n} \int_{\mathbb{R}^{n}} f(y) \sum_{m \in \mathbb{Z}^{n}}\left(\omega^{\beta}\right)^{\vee}(m) \widehat{\varphi}\left(2^{j} y-l+m\right) d y \\
& =2^{j n}\left(f, \Phi^{\beta}\left(2^{j} \cdot-l\right)\right), \quad j \in \mathbb{N},
\end{aligned}
$$

where $\Phi^{\beta}=\Phi_{\mathrm{M}}^{\beta}$ are the mother wavelets of (2.22), (2.23). If $j=0$, then one has to use the father wavelets of (2.21). This completes the proof of parts (ii) and (iii).

\subsection{Proof of Theorem 2}

Step 1. Modifying (2.32) and in analogy to (3.2) we first remark that $\left\{2^{\varepsilon|\beta|} 2^{-j(s-n / p)} \Phi^{\beta}\left(2^{j} x-m\right): j \in \mathbb{N}_{0}, m \in \mathbb{Z}^{n}\right\}, \quad \beta \in \mathbb{N}_{0}^{n}$,

are admitted systems of normalized molecules in $B_{p}^{s}\left(\mathbb{R}^{n}\right)$, provided we ignore constants which are independent of $j, m, \beta$. We refer again to $[2$, Section 5, especially p. 48]. As far as atoms and normalizing factors are concerned one may also consult [13, Theorem 13.8, p. 75]. The proof given there is based on local means and can be extended to molecules (as an alternative argument compared with [2]). In any case the required vanishing moments are covered by (2.19). If $f \in S^{\prime}\left(\mathbb{R}^{n}\right)$ is given by (2.36) with $\left\|\lambda \mid \ell_{p}\right\|_{s}<\infty$ then it now follows in the same way as in Step 1 in 3.1 that $f \in B_{p}^{s}\left(\mathbb{R}^{n}\right)$ and that there is a constant $c>0$ with

$$
\left\|f\left|B_{p}^{s}\left(\mathbb{R}^{n}\right)\|\leq c\| \lambda\right| \ell_{p}\right\|_{s}
$$

for all admitted sequences $\lambda$. To justify the unconditional convergence of (2.36) with $\left\|\lambda \mid \ell_{p}\right\|_{s}<\infty$ in $S^{\prime}\left(\mathbb{R}^{n}\right)$ we apply $\left(\mathrm{id}+(-\Delta)^{L}\right)^{-1}$ with $2 L+s>0$ and $L \in \mathbb{N}$ to $(2.36)$. As will be discussed later on in detail in Subsection 3.4 in connection with the proof of Theorem 3, the resulting sum converges absolutely in $L_{p}\left(\mathbb{R}^{n}\right)$ and hence unconditionally in $S^{\prime}\left(\mathbb{R}^{n}\right)$. But then the original sum (2.36) with $\left\|\lambda \mid \ell_{p}\right\|_{s}<\infty$ converges also unconditionally in $S^{\prime}\left(\mathbb{R}^{n}\right)$. 
Step 2. Let $\left\|k(f) \mid \ell_{p}\right\|_{s}$ with $f \in B_{p}^{s}\left(\mathbb{R}^{n}\right)$ be given by (2.35). We prove that there is a number $c>0$ such that

$$
\left\|k(f)\left|\ell_{p}\left\|_{s} \leq c\right\| f\right| B_{p}^{s}\left(\mathbb{R}^{n}\right)\right\| \quad \text { for all } f \in B_{p}^{s}\left(\mathbb{R}^{n}\right) .
$$

By (2.34), we have

$$
\begin{aligned}
k^{\beta}\left(2^{-j+J}, f\right)\left(2^{-j} m\right) & =\int_{\mathbb{R}^{n}} k^{\beta}(y) f\left(2^{-j} m+2^{-j+J} y\right) d y \\
& =2^{j n-J n} \int_{\mathbb{R}^{n}} k^{\beta}\left(2^{j-J} y-2^{-J} m\right) f(y) d y .
\end{aligned}
$$

Since $\ell_{p}=\left(\ell_{p^{\prime}}\right)^{\prime}$ with $1 / p+1 / p^{\prime}=1$ (hence $\left.1 \leq p^{\prime}<\infty\right)$ and $2^{j n} 2^{j(s-n / p)}=$ $2^{j\left(s+n / p^{\prime}\right)}$ it follows by $(2.35)$ and (3.15) that

$$
\left\|k(f) \mid \ell_{p}\right\|_{s}=\sup \sum_{\beta, j, m} \lambda_{j m}^{\beta} 2^{-J n} \int_{\mathbb{R}^{n}} k^{\beta}\left(2^{j-J} y-2^{-J} m\right) f(y) d y
$$

where the supremum is taken over all $\lambda_{j m}^{\beta}$ such that the terms in (3.16) are non-negative and

$$
\left\|\lambda \mid \ell_{p^{\prime}}\right\|_{-s} \leq 1
$$

Then

$$
\left\|k(f)\left|\ell_{p} \|_{s} \leq \sup \right|(f, g) \mid \text { where } g=2^{-J n} \sum_{\beta, j, m} \lambda_{j m}^{\beta} k^{\beta}\left(2^{j-J} x-2^{-J} m\right)\right.
$$

with (3.17). Hence by Theorem 1,

$$
\left\|k(f) \mid \ell_{p}\right\|_{s} \leq \sup \left\{|(f, g)|: g \in B_{p^{\prime}}^{-s}\left(\mathbb{R}^{n}\right),\left\|g \mid B_{p^{\prime}}^{-s}\left(\mathbb{R}^{n}\right)\right\| \leq c\right\}
$$

for some $c>0$ which is independent of $g$. We use the duality

$$
\left(B_{p^{\prime}}^{-\sigma}\left(\mathbb{R}^{n}\right)\right)^{\prime}=B_{p}^{\sigma}\left(\mathbb{R}^{n}\right), \quad 1 \leq p^{\prime}<\infty, \sigma \in \mathbb{R},
$$

which can be found in [11, 2.11.2, p. 178]. Now, (3.14) is a consequence of (3.18), (3.19).

Step 3. We prove parts (ii) and (iii) of the theorem. Then we get as a by-product the converse of (3.13) and hence part (i). Let $1<p \leq \infty, s<0$, and $f \in B_{p}^{s}\left(\mathbb{R}^{n}\right)$. We apply Theorem 1 to $\psi \in S\left(\mathbb{R}^{n}\right)$,

$$
\psi=\sum_{\beta, j, m} 2^{j n}\left(\psi, \Phi^{\beta}\left(2^{j} \cdot-m\right)\right) k^{\beta}\left(2^{j-J} x-2^{-J} m\right),
$$

to obtain

$$
(f, \psi)=\left(\sum_{\beta, j, m} 2^{j n}\left(k^{\beta}\left(2^{j-J} \cdot-2^{-J} m\right), f\right) \Phi^{\beta}\left(2^{j} \cdot-m\right), \psi\right) .
$$

Since $\psi \in S\left(\mathbb{R}^{n}\right)$ it follows by Theorem 1 that (3.20) converges unconditionally in any space $B_{p^{\prime}}^{\sigma}\left(\mathbb{R}^{n}\right)$ with $\sigma>0$. This justifies (3.21). Then (2.37) is a consequence of (3.21) and (3.15). Since (3.20) converges unconditionally 
in any space $B_{p^{\prime}}^{\sigma}\left(\mathbb{R}^{n}\right)$ it follows that (2.37) also converges unconditionally in $S^{\prime}\left(\mathbb{R}^{n}\right)$. This proves the first assertion in part (ii). Furthermore we have the representation (2.37) in particular for $f \in B_{p}^{s}\left(\mathbb{R}^{n}\right)$. Then (3.13) and (3.14) prove part (iii) and the second assertion in part (ii).

\subsection{Proof of Corollary 1}

Step 1. We begin with some preparations. Let, as usual,

$$
\langle x\rangle=\left(1+|x|^{2}\right)^{1 / 2}, \quad x \in \mathbb{R}^{n} .
$$

Let $\alpha \in \mathbb{N}_{0}^{n}, \varrho>0$, and $d>0$ be given. Then we wish to show that there is a constant $c=c(\alpha, \varrho, d)$ such that

$$
\left|D^{\alpha} \Phi^{\beta}(x)\right| \leq c 2^{-\varrho|\beta|}\langle x\rangle^{-d}, \quad x \in \mathbb{R}^{n}, \beta \in \mathbb{N}_{0}^{n},
$$

where $\Phi^{\beta}$ is either $\Phi_{\mathrm{F}}^{\beta}$ or $\Phi_{\mathrm{M}}^{\beta}$ defined by (2.21) or (2.22), respectively. By (2.15), (3.11) it follows that

$$
\left|\left(\omega^{\beta}\right)^{\vee}(y)\right| \leq c 2^{-\kappa|\beta|}\left|D^{\beta} \omega^{\vee}(y)\right| \leq c 2^{-\varrho|\beta|}\langle y\rangle^{-a}, \quad y \in \mathbb{R}^{n},
$$

where $\kappa>0$, and hence $\varrho>0$, and $a>0$, are at our disposal. Consequently, we have

$$
\left|D^{\alpha} \Phi^{\beta}(x)\right| \leq c 2^{-\varrho|\beta|} \sum_{m \in \mathbb{Z}^{n}} \frac{1}{\langle m\rangle^{a}\langle x+m\rangle^{a}} \leq c 2^{-\varrho|\beta|}\langle x\rangle^{-d}, \quad x \in \mathbb{R}^{n} .
$$

where $\varrho>0$ and $a>0$, and hence $d>0$, are at our disposal. This proves (3.22) where $c$ depends on $\varrho, d, \alpha$, but not on $\beta$ and $x$.

Step 2. Let $f \in B_{p}^{s}\left(\mathbb{R}^{n}\right)$. We fix $\beta \in \mathbb{N}_{0}^{n}$ and $j \in \mathbb{N}_{0}$ in the sum in (2.37) and denote the resulting sum over $m \in \mathbb{Z}^{n}$ by $f_{\beta, j}$. It follows by (2.38), (2.35) and (3.22) that

$$
\begin{aligned}
\left|D^{\alpha} f_{\beta, j}(x)\right| & =2^{J n}\left|\sum_{m \in \mathbb{Z}^{n}} k^{\beta}\left(2^{-j+J}, f\right)\left(2^{-j} m\right)\left(D^{\alpha} \Phi^{\beta}\right)\left(2^{j} x-m\right) 2^{j|\alpha|}\right| \\
& \leq c 2^{-j(s-n / p)+j|\alpha|} 2^{-\varrho|\beta|} \sum_{m \in \mathbb{Z}^{n}}\left\langle 2^{j} x-m\right\rangle^{-d},
\end{aligned}
$$

where $d>0$ and $\varrho>0$ are at our disposal. Then we conclude that $f_{\beta, j}$ and also $f_{j}$ with $j \in \mathbb{N}_{0}$, where

$$
f_{j}(x)=\sum_{\beta \in \mathbb{N}_{0}^{n}} f_{\beta, j}(x) \quad \text { are } C^{\infty} \text { functions in } \mathbb{R}^{n} .
$$

Step 3. We prove (2.44). We may assume $x^{0}=0$. By (2.37), (2.41) the remainder function can be written as

$$
f-f^{K, x^{0}}=\sum_{\beta, j} f_{\beta, j}^{K}
$$


with

$$
f_{\beta, j}^{K}(x)=2^{J n} \sum_{m \in \mathbb{Z}^{n}}^{K} k^{\beta}\left(2^{-j+J}, f\right)\left(2^{-j} m\right) \Phi^{\beta}\left(2^{j} x-m\right),
$$

where by (2.42) the summation in (3.26) is restricted to $m \in \mathbb{Z}^{n}$ with

$$
\left|2^{-j} m-2^{-j+J}\right| \geq 2^{-K+1} .
$$

By Step 2 we may assume $j>J+K$. Then we have

$$
|m| \geq 2^{j-K} \text {. }
$$

We restrict the summation in (3.23) to $m \in \mathbb{Z}^{n}$ with (3.27). If $|x| \leq 2^{-K-1}$ then $\left|m-2^{j} x\right| \geq 2^{j-K-1}$. Then it follows as in (3.23) that

$$
\left|D^{\alpha} f_{\beta, j}^{K}(x)\right| \leq c 2^{-j(s-n / p)+j|\alpha|} 2^{-\varrho|\beta|} 2^{-j d}, \quad|x| \leq 2^{-K-1},
$$

where $d>0$ is at our disposal. Now we are in the same position as in Step 2 and there is an obvious counterpart of (3.24). In the ball $B\left(0,2^{-K-1}\right)$ we in addition have (3.28). Then it follows by summation over $\beta \in \mathbb{N}_{0}^{n}$ and $j \in \mathbb{N}_{0}$ that $f-f^{K, x^{0}}$ in $(3.25)$ is a $C^{\infty}$ function in $B\left(0,2^{-K-1}\right)$.

Step 4. We proved (2.45) in Remark 6 after Corollary 1 where $\sigma<0$ is not necessary. We now prove (2.46). Let

$$
f^{K, x^{0}} \in B_{p}^{\sigma}\left(\mathbb{R}^{n}\right), \quad 1<p \leq \infty, s \leq \sigma<0 .
$$

By Remark 5 and the notation (2.35) we have

$$
\left\|k\left(f^{K, x^{0}}\right)\left|\ell_{p}\left\|_{\sigma} \leq c\right\| f^{K, x^{0}}\right| B_{p}^{\sigma}\left(\mathbb{R}^{n}\right)\right\|<\infty .
$$

Let again $x^{0}=0$. By (2.44), for $|x| \leq 2^{-K-2}$ we have

$$
f(x)=f^{K, x^{0}}(x)+g(x) \quad \text { with } \quad g \in C_{0}^{\infty}\left(B\left(0,2^{-K-1}\right)\right) .
$$

We can apply (3.29) to $g$ as an element of $B_{p}^{\kappa}\left(\mathbb{R}^{n}\right)$ with $\sigma \leq \kappa<0$ and obtain

$$
\left\|k(g) \mid \ell_{p}\right\|_{\kappa}<\infty .
$$

To calculate the right-hand side of (2.46) we assume, as in (2.42),

$$
B\left(0,2^{-K-2}\right) \cap B\left(2^{-j} m, 2^{-j+J}\right) \neq \emptyset
$$

for some $j>K+J+2$ and $m \in \mathbb{Z}^{n}$. This is sufficient since $f \in B_{p}^{s}\left(\mathbb{R}^{n}\right)$ and, hence, there is nothing to prove for the terms with $j \leq K+J+2$. Then, by (2.34), (3.30),

$$
k^{\beta}\left(2^{-j+J}, f\right)\left(2^{-j} m\right)=k^{\beta}\left(2^{-j+J}, f^{K, x^{0}}\right)\left(2^{-j} m\right)+k^{\beta}\left(2^{-j+J}, g\right)\left(2^{-j} m\right) .
$$

Now (2.46) follows from (3.29), (3.31). The proof of Corollary 1 is complete. 


\subsection{Proof of Theorem 3}

Step 1. Let $f$ be given by (2.58) with $\left\|\lambda \mid \ell_{p}\right\|_{s}<\infty$. By (2.56) this is a molecular representation, and again it follows that $f \in B_{p}^{s}\left(\mathbb{R}^{n}\right)$ and

$$
\left\|f\left|B_{p}^{s}\left(\mathbb{R}^{n}\right)\|\leq c\| \lambda\right| \ell_{p}\right\|_{s},
$$

where $c$ is independent of $\lambda$. The absolute convergence of $(2.58)$ in $L_{p}\left(\mathbb{R}^{n}\right)$ is again a consequence of our considerations in [14, 1.4], appropriately modified.

Step 2. We prove part (ii). Then we get as a by-product also the converse of (3.32) and hence part (i). Since $s-2 L<0$ we have by Theorem 2 the optimal representation (2.49). First we prove that

$$
k^{\beta}\left(2^{-j+J}, D_{L} f\right)\left(2^{-j} m\right)=2^{2 L(j-J)} \lambda_{j m}^{\beta}(f),
$$

where $\lambda_{j m}^{\beta}(f)$ is given by (2.61). This follows from

$$
\begin{aligned}
& k^{\beta}\left(2^{-j+J}, D_{L} f\right)(x) \\
& \quad=k^{\beta}\left(2^{-j+J}, f\right)(x)+\int_{\mathbb{R}^{n}} k^{\beta}(y)\left[(-\Delta)^{L} f\right]\left(x+2^{-j+J} y\right) d y \\
& \quad=k^{\beta}\left(2^{-j+J}, f\right)(x)+2^{2 L(j-J)} k_{L}^{\beta}\left(2^{-j+J}, f\right)(x), \quad x \in \mathbb{R}^{n},
\end{aligned}
$$

where we used the notation (2.57). Since the coefficients in (3.33) are optimal for $D_{L} f \in B_{p}^{s-2 L}\left(\mathbb{R}^{n}\right)$ it follows by Theorem 2 that

$$
\begin{aligned}
\left\|\lambda(f) \mid \ell_{p}\right\|_{s} & =2^{2 L J}\left\|k\left(D_{L} f\right) \mid \ell_{p}\right\|_{s-2 L} \\
& \sim\left\|D_{L} f\left|B_{p}^{s-2 L}\left(\mathbb{R}^{n}\right)\|\sim\| f\right| B_{p}^{s}\left(\mathbb{R}^{n}\right)\right\| .
\end{aligned}
$$

Hence, by (2.49) we have the decomposition

$$
f=2^{J(n-2 L)} \sum_{\beta, j, m} \lambda_{j m}^{\beta}(f) 2^{2 j L} D_{L}^{-1}\left[\Phi^{\beta}\left(2^{j} \cdot-m\right)\right](x) .
$$

The proof that this representation coincides with (2.60) is postponed to the next step. We justify (2.62), where the first equivalence is covered by (3.35). Furthermore, by (3.34), (3.35),

$$
\begin{aligned}
\left\|f \mid B_{p}^{s}\left(\mathbb{R}^{n}\right)\right\| & \leq c_{1}\left\|k_{L}(f)\left|\ell_{p}\left\|_{s}+c_{1}\right\| k(f)\right| \ell_{p}\right\|_{s-2 L} \\
& \leq c_{2}\left\|k_{L}(f)\left|\ell_{p}\left\|_{s}+c_{2}\right\| f\right| B_{p}^{s-2 L}\left(\mathbb{R}^{n}\right)\right\| \\
& \leq c_{3}\left\|k_{L}(f)\left|\ell_{p}\left\|_{s}+c_{3}\right\| f\right| L_{p}\left(\mathbb{R}^{n}\right)\right\|,
\end{aligned}
$$

where we used Theorem 2 and $s-2 L<0$. Conversely, again by (3.34), (3.35), we have

$$
\begin{aligned}
\left\|k_{L}(f)\left|\ell_{p}\left\|_{s}+\right\| f\right| L_{p}\left(\mathbb{R}^{n}\right)\right\| & \leq c\left\|f\left|B_{p}^{s}\left(\mathbb{R}^{n}\right)\|+c\| f\right| L_{p}\left(\mathbb{R}^{n}\right)\right\| \\
& \leq c^{\prime}\left\|f \mid B_{p}^{s}\left(\mathbb{R}^{n}\right)\right\| .
\end{aligned}
$$

This proves (2.62). 
Step 3. By (3.36) and (2.60) it remains to prove that (3.37)

$$
2^{2 j L} D_{L}^{-1}\left[\Phi^{\beta}\left(2^{j} \cdot-m\right)\right](x)=\left(\Phi^{\beta, L}+2^{-2 j L} \Phi_{j}^{\beta, L}\right)\left(2^{j} x-m\right),
$$

where the wavelets $\Phi^{\beta, L}$ and $\Phi_{j}^{\beta, L}$ are given by (2.50)-(2.52) and (2.54). Let $j=0$. Then $\Phi^{\beta, L}=\Phi_{\mathrm{F}}^{\beta, L}$ are the father wavelets. By $(2.17)$ we have

$$
F^{-1}\left[\left(\mathrm{id}+(-\Delta)^{L}\right)^{-1} \Phi_{\mathrm{F}}^{\beta}(\cdot-m)\right](\xi)=\frac{\varphi_{0}(\xi) \Omega^{\beta}(\xi)}{1+|\xi|^{2 L}} e^{i m \xi} .
$$

Taking the Fourier transform we get the desired assertion

$$
D_{L}^{-1}\left[\Phi_{\mathrm{F}}^{\beta}(\cdot-m)\right](x)=\Phi_{\mathrm{F}}^{\beta, L}(x-m), \quad x \in \mathbb{R}^{n}, m \in \mathbb{Z}^{n} .
$$

Let $j \in \mathbb{N}$. Then $\Phi^{\beta, L}=\Phi_{\mathrm{M}}^{\beta, L}$ are the mother wavelets. By (2.18) and (2.51) we have

$$
\begin{aligned}
2^{2 j L} F^{-1}\left[(-\Delta)^{-L} \Phi_{\mathrm{M}}^{\beta}\left(2^{j} \cdot-m\right)\right](\xi) & =\frac{2^{-j n}}{\left|2^{-j} \xi\right|^{2 L}} F^{-1}\left[\Phi_{\mathrm{M}}^{\beta}(\cdot-m)\right]\left(2^{-j} \xi\right) \\
& =2^{-j n} e^{i 2^{-j} m \xi} F^{-1}\left[\Phi_{\mathrm{M}}^{\beta, L}\right]\left(2^{-j} \xi\right) .
\end{aligned}
$$

Taking the Fourier transform we get

$$
2^{2 j L}(-\Delta)^{-L}\left[\Phi_{\mathrm{M}}^{\beta}\left(2^{j} \cdot-m\right)\right](x)=\Phi_{\mathrm{M}}^{\beta, L}\left(2^{j} x-m\right) .
$$

Similarly by (2.52) for the remainder term we have

$$
\begin{aligned}
2^{2 j L} F^{-1}[((\mathrm{id} & \left.\left.\left.+(-\Delta)^{L}\right)^{-1}-(-\Delta)^{-L}\right) \Phi_{\mathrm{M}}^{\beta}\left(2^{j} \cdot-m\right)\right](\xi) \\
& =2^{-j n} 2^{2 j L}\left(\frac{1}{1+|\xi|^{2 L}}-\frac{1}{|\xi|^{2 L}}\right) e^{i 2^{-j} m \xi} F^{-1}\left[\Phi_{\mathrm{M}}^{\beta}\right]\left(2^{-j} \xi\right) \\
& =-2^{-j n} e^{i 2^{-j} m \xi} \frac{2^{-2 j L}}{\left|2^{-j} \xi\right|^{2 L}\left(2^{-2 j L}+\left|2^{-j} \xi\right|^{2 L}\right)} F^{-1}\left[\Phi_{\mathrm{M}}^{\beta}\right]\left(2^{-j} \xi\right) \\
& =2^{-2 j L} 2^{-j n} e^{i 2^{-j} m \xi} F^{-1}\left[\Phi_{j}^{\beta, L}\right]\left(2^{-j} \xi\right) .
\end{aligned}
$$

Taking the Fourier transform we get

$$
\begin{aligned}
& 2^{2 j L}\left[\left(\mathrm{id}+(-\Delta)^{L}\right)^{-1}-(-\Delta)^{-L}\right]\left[\Phi_{\mathrm{M}}^{\beta}\left(2^{j} \cdot-m\right)\right](x) \\
&= 2^{-2 j L} \Phi_{j}^{\beta, L}\left(2^{j} x-m\right) .
\end{aligned}
$$

Now, (3.37) is a consequence of (3.38)-(3.40).

3.5. Proof of Corollary 2. We discussed in Remark 7 the properties of the wavelets in the representations (2.60), (2.65), (2.66). Then the arguments from Steps 1-3 of the proof of Corollary 1 in 3.3 can be applied. This proves (2.67). Furthermore, $\widetilde{f}_{L}^{K, x^{0}}$ and $f_{L}^{K, x^{0}}$ differ by local versions of the functions $f^{\prime}$ and $f^{\prime \prime}$ in 2.4.3. Then (2.68) follows from (2.67). Finally, (2.69) is a consequence of the molecular representation (2.66); and (2.70) follows from (2.68) in the same way as in Step 4 of 3.3. 


\subsection{Proof of Theorem 4}

Step 1. First we assume that there is no $L \in \mathbb{N}_{0}$ such that (2.72) holds for some $\varepsilon>0$ and all $K \in \mathbb{N}$. Hence for all $L \in \mathbb{N}_{0}$ and all $\varepsilon>0$, there is a $K \in \mathbb{N}$ with

$$
\left\|k_{L}(f) \mid \ell_{p}\right\|_{2 L-\varepsilon, K, x^{0}}<\infty .
$$

Recall $k(f)=k_{0}(f)$. Then by (2.74) we have

$$
\left\|k(f)\left|\ell_{p}\left\|_{\sigma-2 L, K, x^{0}}+\right\| k_{L}(f)\right| \ell_{p}\right\|_{\sigma, K, x^{0}}<\infty
$$

for any $\sigma<2 L$ and all $K \geq K_{0}$. Hence by (2.67) and the molecular representation (2.65) with (2.61) it follows that

$$
f \in B_{p}^{\sigma}\left(B\left(x^{0}, 2^{-K-1}\right)\right), \quad 0<\sigma<2 L,
$$

for any $L \in \mathbb{N}$ and any $\sigma$. Hence $s_{f}\left(x^{0}, t\right)=\infty$ by Definition 4 . Now we assume $s_{f}\left(x^{0}, t\right)=\infty$. Then we deduce by Definition 4 and Corollary 2 that there is no $L \in \mathbb{N}_{0}$ with (2.72) for some $\varepsilon>0$ and all $K \in \mathbb{N}$. This proves the first assertion of Theorem 4.

Step 2. Let now $s_{f}\left(x^{0}, t\right)<\infty$ and $L_{0}=L\left(f, x^{0}\right) \in \mathbb{N}_{0}$ be the smallest number with (2.72) for some $\varepsilon>0$ and all $K \in \mathbb{N}$. Let

$$
\begin{aligned}
& \widetilde{s}_{f}\left(x^{0}, t\right) \\
& =\inf \left\{2 L_{0}-\varepsilon: \varepsilon>0,\left\|k_{L_{0}}(f) \mid \ell_{p}\right\|_{2 L_{0}-\varepsilon, K, x^{0}}=\infty \text { for all } K \in \mathbb{N}\right\} .
\end{aligned}
$$

We check that

$$
-\infty<\widetilde{s}_{f}\left(x^{0}, t\right)<\infty .
$$

The existence of $L_{0}$, and hence the right-hand inequality of (3.42), follow from Step 1. On the other hand, by assumption we have $f \in B_{p}^{s}\left(\mathbb{R}^{n}\right)$ for some $s<0$. If $L_{0}=0$, then by Corollary 1 ,

$$
-\infty<s \leq \widetilde{s}_{f}\left(x^{0}, t\right), \quad L_{0}=0 .
$$

Let $L_{0} \in \mathbb{N}$. Again by Corollary $1, f^{K, x^{0}} \in B_{p}^{\sigma}\left(\mathbb{R}^{n}\right)$ for any $\sigma<0$. Furthermore by lifting arguments of type $(3.34)$ (with respect to $B_{p}^{\sigma-2 L_{0}}\left(\mathbb{R}^{n}\right)$ ) it follows that

$$
\left\|k_{L_{0}}(f) \mid \ell_{p}\right\|_{\sigma, K, x^{0}}<\infty \quad \text { for all } K \in \mathbb{N} .
$$

In other words we obtain, by (3.41),

$$
0 \leq \widetilde{s}_{f}\left(x^{0}, t\right), \quad L_{0} \in \mathbb{N} .
$$

Thus we have the left-hand inequality of (3.42) in all cases.

Step 3. Let again $s_{f}\left(x^{0}, t\right)<\infty$ and $L_{0}=0$. If $\sigma<\widetilde{s_{f}}\left(x^{0}, t\right)<0$ then $f^{K, x^{0}} \in B_{p}^{\sigma}\left(\mathbb{R}^{n}\right)$ by (2.45). Hence

$$
\widetilde{s}_{f}\left(x^{0}, t\right) \leq s_{f}\left(x^{0}, t\right) .
$$


Conversely, if $\sigma<s_{f}\left(x^{0}, t\right)$ then $\left\|k(f) \mid \ell_{p}\right\|_{\sigma, K+3, x^{0}}<\infty$ by (2.46), and hence

$$
s_{f}\left(x^{0}, t\right) \leq \widetilde{s}_{f}\left(x^{0}, t\right)<0 .
$$

This proves

$$
s_{f}\left(x^{0}, t\right)=\widetilde{s}_{f}\left(x^{0}, t\right)
$$

in case $L_{0}=0$ in (3.41).

Step 4. Let again $s_{f}\left(x^{0}, t\right)<\infty$ and $L_{0} \in \mathbb{N}$. By Corollary 1 and (3.43) we have

$$
s_{f}\left(x^{0}, t\right) \geq 0 \quad \text { and } \quad \tilde{s}_{f}\left(x^{0}, t\right) \geq 0 .
$$

If $s_{f}\left(x^{0}, t\right)>0$ then we can apply Corollary 2 to obtain (3.44) again. Conversely, let $\widetilde{s}_{f}\left(x^{0}, t\right)>0$. Here we must add a technical comment: The a priori assumption $f \in B_{p}^{s}\left(\mathbb{R}^{n}\right)$ with $s>0$ in Corollary 2 can be replaced by $f \in B_{p}^{\sigma}\left(\mathbb{R}^{n}\right)$ for all $\sigma<0$. This follows from the proof in 3.5 and the references given there (we just used this type of assumption). Then we can again apply Corollary 2 to obtain (3.44).

Step 5. Hence if one of the numbers in (3.44) is negative, or positive, then so is the other, and they coincide. But then they must also coincide if one of the numbers is zero. Finally, we remark that (3.41) coincides with the right-hand side of $(2.73)$.

3.7. Proof of Proposition 2. We prove part (i). Let $k$ and $k^{\beta}$ be given by (2.7)-(2.10). Then (2.78) is satisfied uniformly for all functions $2^{\varepsilon|\beta|} k^{\beta}(x)$ in place of $k$, where $\varepsilon>0$ is some number (since $\operatorname{supp} k$ is in $B\left(0,2^{-\varepsilon}\right)$ ). Hence by the left-hand assumption of (2.79),

$$
\left(\sum_{j, m}^{K, x^{0}} 2^{j(\sigma-n / p) p}\left|k^{\beta}\left(2^{-j+J}, f\right)\left(2^{-j} m\right)\right|^{p}\right)^{1 / p} \leq c 2^{-\varepsilon|\beta|}
$$

for some $c>0$ which is independent of $\beta$. Then $f^{K, x^{0}} \in B_{p}^{\sigma}\left(\mathbb{R}^{n}\right)$ follows from (2.43) with $\sigma$ in place of $s$ and (2.45). The proof of part (ii) is the same, now based on (2.71), (2.69).

\section{References}

[1] I. Daubechies, Ten Lectures on Wavelets, CBMS-NSF Regional Conf. Ser. in Appl. Math. 61, SIAM, Philadelphia, 1992.

[2] M. Frazier, B. Jawerth and G. Weiss, Littlewood-Paley Theory and the Study of Function Spaces, CBMS Regional Conf. Ser. in Math. 79, Amer. Math. Soc., Providence, 1991.

[3] S. Jaffard, On the Frisch-Parisi conjecture, J. Math. Pures Appl. 79 (2000), 525-552. 
[4] S. Jaffard, Wavelet expansions, function spaces and multifractal analysis, in: Twentieth Century Harmonic Analysis-A Celebration (Il Ciocco, 2000), Kluwer, Dordrecht, 2001, 127-144.

[5] S. Jaffard and Y. Meyer, Wavelet methods for pointwise regularity and local oscillations of functions, Mem. Amer. Math. Soc. 587 (1996).

[6] S. Mallat, A Wavelet Tour of Signal Processing, Academic Press, San Diego, 1999.

[7] Y. Meyer, Wavelets and Operators, Cambridge Univ. Press, Cambridge, 1992.

[8] -, Wavelets, vibrations and scalings, CRM Monogr. Ser. 9, Amer. Math. Soc., Providence, 1998.

[9] T. Runst and W. Sickel, Sobolev Spaces of Fractional Order, Nemytskij Operators, and Nonlinear Partial Differential Equations, de Gruyter, Berlin, 1996.

[10] H. Triebel, Fourier Analysis and Function Spaces, Teubner, Leipzig, 1977.

[11] —, Theory of Function Spaces, Birkhäuser, Basel, 1983.

[12] -, Theory of Function Spaces II, Birkhäuser, Basel, 1992.

[13] - Fractals and Spectra, Birkhäuser, Basel, 1997.

[14] —, The Structure of Functions, Birkhäuser, Basel, 2001.

[15] - Towards a Gausslet analysis: Gaussian representations of functions, in: Function Spaces. Interpolation Theory and Related Topics (Lund, 2000), de Gruyter, Berlin, 2002, 425-449.

[16] - Fractal characteristics of measures, an approach via function spaces, Jena, 2001 (submitted).

[17] H. Winkelvoss, Function spaces related to fractals. Intrinsic atomic characterizations of function spaces on domains, $\mathrm{PhD}$ thesis, Jena, 1995.

[18] P. Wojtaszczyk, A Mathematical Introduction to Wavelets, Cambridge Univ. Press, Cambridge, 1997.

Mathematisches Institut

Fakultät für Mathematik und Informatik

Friedrich-Schiller-Universität Jena

D-07740 Jena, Germany

E-mail: triebel@minet.uni-jena.de 